\title{
Optimal fitting of gaussian-apodized or under-resolved emission lines in Fourier Transform spectra providing new insights on the velocity structure of NGC 6720
}

\author{
Thomas B. Martin, ${ }^{1 \star ~ S i m o n ~ P r u n e t, ~}{ }^{2}$ Laurent Drissen, ${ }^{1}$ \\ ${ }^{1}$ Université Laval, 2325, rue de l'université, Québec (Québec), G1V 0A6, Canada \\ ${ }^{2}$ Canada-France-Hawaii Telescope, 65-1238 Mamalahoa Hwy, Kamuela, Hawaii 96743, USA
}

Accepted XXX. Received YYY; in original form ZZZ

\begin{abstract}
An analysis of the kinematics of NGC 6720 is performed on the commissioning data obtained with SITELLE, the Canada-France-Hawaii Telescope's new imaging Fourier transform spectrometer. In order to measure carefully the small broadening effect of a shell expansion on an unresolved emission line, we have determined a computationally robust implementation of the convolution of a Gaussian with a sinc instrumental line shape which avoids arithmetic overflows. This model can be used to measure line broadening of typically a few $\mathrm{km} \mathrm{s}^{-1}$ even at low spectral resolution (R less than 5000). We have also designed the corresponding set of Gaussian apodizing functions that are now used by ORBS, the SITELLE's reduction pipeline. We have implemented this model in ORCS, a fitting engine for SITELLE's data, and used it to derive the [S II] density map of the central part of the nebula. The study of the broadening of the [N II] lines shows that the Main Ring and the Central Lobe are two different shells with different expansion velocities. We have also derived deep and spatially resolved velocity maps of the Halo in $[\mathrm{NII}]$ and $\mathrm{H} \alpha$ and found that the brightest bubbles are originating from two bipolar structures with a velocity difference of more than $35 \mathrm{~km} \mathrm{~s}^{-1}$ lying at the poles of a possibly unique Halo shell expanding at a velocity of more than $15 \mathrm{~km} \mathrm{~s}^{-1}$.
\end{abstract}

Key words: instrumentation: spectrographs - methods: data analysis - methods: numerical - techniques: imaging spectroscopy - planetary nebulae: individual: M57 ISM: kinematics and dynamics

\section{INTRODUCTION}

The high quality of the data obtained during the recent commissioning of SITELLE (Drissen et al. 2010), the CanadaFrance-Hawaii Telescope (CFHT)'s new imaging Fourier transform spectrometer, (Baril et al. (2016), Drissen et al., in preparation) revealed that the model of the emission lines used for the analysis of SITELLE's data with its dedicated software, ORCS (Martin et al. 2015), was not perfectly suitable because the original shape of the observed lines was not taken into account in the model. Only the instrumental line shape (ILS) was considered. We have thus designed a new line model resulting from the convolution of a Gaussian line profile with the ILS which could be used to measure very small broadening values (typically a few $\mathrm{km} \mathrm{s}^{-1}$ ) even at low spectral resolution ( $\mathrm{R}$ less than 5000). In Section 2, we describe our new line model and determine a more computa-

* E-mail: thomas.martin.1@ulaval.ca tionally robust implementation for small broadening values which avoids arithmetic overflows. In Section 3, we provide the definition of a set of Gaussian apodizing functions with an adjustable broadening parameter that is perfectly suitable for this model. We then apply this new line model to a data cube of the planetary nebula NGC 6720 (the Ring nebula, M 57), which was SITELLE's first target during commissioning. This analysis provides new insights on its velocity structure which are then presented in section 4 .

\section{DESIGN OF A COMPUTATIONALLY ROBUST MODEL FOR A SINC LINE WITH GAUSSIAN BROADENING}

\subsection{The instrumental line shape of a Fourier transform spectrum}

Although dispersive spectroscopy is, by far, the most widely used technique to obtain spectra of astronomical targets, 
a)

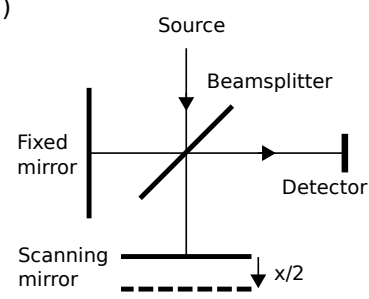

b)

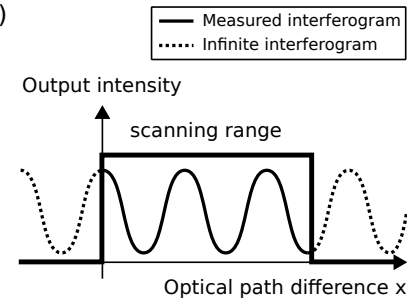

Figure 1. a) Sketch of a classical Michelson interferometer. When the scanning mirror is moved from a distance $x / 2$ from the position where the two interfering beams have the same optical path, the OPD is equal to $x$. b) Ideal interferogram of a monochromatic source (a cosine). We also show the real scanning range represented as a rectangular function and the infinite interferogram which could be recorded if the scanning range was infinite.

Fourier transform spectrometers (FTS) have been successfully used, both on ground-based telescopes and space observatories: SPIRE on Herschel (Griffin et al. 2010), FISFTS on AKARI (Kawada et al. 2008), PFS on Mars Express (Formisano et al. 2005) and CIRS on the Cassini orbiter (Kunde et al. 1996), BEAR at CFHT (Maillard \& Michel 1982), FTS-2 at the James Clerk Maxwell Telescope (Naylor et al. 2006), all working at infrared and sub$\mathrm{mm}$ wavelengths. Our team has also developped SpIOMM, SITELLE's prototype, at the Observatoire du Mont Mégantic (Grandmont 2003; Bernier 2006). In all cases, the core of the instrument is a Michelson interferometer, which acts exactly like an inverse Fourier transform on the input light and the signal sampled at its output is an interferogram. To recover the input signal's spectrum, one has to apply a Fourier transform to the measured interferogram. Combining the interferometer with imaging optics and a detector array like a bolometer array or a charge-coupled device (CCD), millions of spatially resolved interferograms of extended sources can be recorded simultaneously by these imaging Fourier transform spectrometers (iFTS). SPIOMM and SITELLE have pushed this concept to the more technically challenging near-UV and visible wavelengths (350 - $800 \mathrm{~nm})$, wide fields of view $\left(11^{\prime} \times 11^{\prime}\right)$, and spaxel numbers (4 million for SITELLE).

Although SITELLE uses an off-axis configuration to allow the recording of both output ports, the example of a classical Michelson interferometer can be used to show how an interferogram is recorded (see Fig. 1). The collimated beam at the input is divided into two (ideally identical) parts by the beamsplitter. The two beams are then reflected back onto the beamsplitter by two mirrors (one is fixed while the other can be moved) where they interfere, giving two complementary output beams: one is going back to the source while the other can eventually be measured by a detector. During a scan, the optical path difference (OPD, noted $x$ and measured in $\mathrm{cm}$ ) between the two interfering beams is gradually changed, step by step, modulating the output intensity according to the spectral energy distribution of the observed source. The intensity recorded at each step forms the interferogram; its Fourier transform must be computed to recover the spectrum of the source.

One of the most distinctive features of a spectrum ob-

tained with an FTS is the ILS which is a sinc ${ }^{1}$. It comes from the fact that the number of observed samples is finite thus implying a finite maximum value of the OPD. Any measured interferogram $I(x)$ can thus be considered as an infinite interferogram $I_{\infty}(x)$ multiplied with a rectangular function $\Pi(x)$ :

$I(x)=I_{\infty}(x) \Pi(x)$

As the spectrum is the Fourier transform of the interferogram, the convolution theorem states that the spectrum will be the convolution of the real spectrum at an infinite resolution with the Fourier transform of a rectangular function, i.e. a pure sinc function.

$$
\begin{aligned}
S(\sigma) & =T F[I(x)]=T F\left[I_{\infty}(x)\right] * T F[\Pi(x)] \\
& =S_{\mathrm{R}=\infty}(\sigma) * \operatorname{sinc}(\sigma) .
\end{aligned}
$$

If the spectrum is a monochromatic line of flux unity and wavenumber $\sigma_{0}$, its spectrum is similar to a Dirac deltafunction $\delta\left(\sigma-\sigma_{0}\right)$ and the resulting measured spectral line $S_{\sigma_{0}}(\sigma)$ is, by definition, the instrumental line shape.

$$
S_{\sigma_{0}}(\sigma)=\delta\left(\sigma-\sigma_{0}\right) * \operatorname{sinc}(\sigma)=\operatorname{sinc}\left(\sigma-\sigma_{0}\right)
$$

The ILS of an ideal FTS can fully be described via its full-width at half-maximum (FWHM) which limits the resolution of the convoluted spectrum and depends only on the maximum OPD (MPD) reached during the interferogram recording. The equation giving the width of the sinc, $\Delta w$ (in $\mathrm{cm}^{-1}$ ), from the MPD of the interferogram (in $\mathrm{cm}$ ), can be written as (Kauppinen \& Partanen 2001)

$\Delta w=\frac{1}{2 \mathrm{MPD}}$,

with the FWHM of the sinc function, FWHM $=1.20671 \Delta w$ (see equation 9). At a given wavenumber $\sigma$ (in $\mathrm{cm}^{-1}$ ), the resolution $R$ is therefore (Martin 2015):

$R=\frac{\sigma}{\mathrm{FWHM}}=\frac{\sigma}{1.20671 \Delta w}=\frac{2 \sigma \mathrm{MPD}}{1.20671}$.

On the optical axis of the interferometer, the MPD is the product of the optical step size, $\delta_{x}$, by the number of steps, $N$, on the largest side of the interferogram with respect to the zero path difference. But if we are recording an interferogram at a certain angle $\theta$ a cosine term must be taken into account.

$\mathrm{MPD}=N \delta_{x} \cos \theta$

The knowledge of the ILS is very important if we want to fit emission and absorption lines in a Fourier transform spectrum (Martin et al. 2015). Even if we know that the model of a pure monochromatic line is a sinc (with an ideal instrument), perfectly monochromatic lines do not exist. Their shape is mostly determined by their emission mechanism (internal motions in an HII region, for instance). Therefore, the observed line shape is always the convolution of the ILS with the intrinsic line shape as it ends up on our detectors.

1 Throughout this paper the sinc function is defined as $\operatorname{sinc}(\sigma)=$ $\frac{\sin (\pi \sigma)}{\pi \sigma}$. 


\subsection{General model of a sinc ILS with Gaussian broadening}

Due to thermal Doppler broadening, star rotation or gas motion broadening, the vast majority of the observed lines, in emission as well as in absorption, have a Gaussian shape. At low resolutions $(R<1000)$, the width of the line is generally small enough compared with the width of the ILS that a pure sinc function can be considered as a good model for line fitting. But, as we will see in this section, at higher resolution or when the line broadening is sufficiently high, a line will start to exhibit a larger profile which is better modelized as the convolution of a sinc, $S_{\Delta w}\left(\sigma-\sigma_{0}\right)$, of width $\Delta w$ centered on the wavenumber of the line $\left(\sigma_{0}\right)$ and a Gaussian, $G_{\Delta \sigma}(\sigma)$, of width $\Delta \sigma$,

$S G(\sigma)=S_{\Delta w}(\sigma) * \delta\left(\sigma-\sigma_{0}\right) * G_{\Delta \sigma}(\sigma)$,

with,

$S_{\Delta w}(\sigma)=\operatorname{sinc}\left(\frac{\sigma}{\pi \Delta w}\right)=\frac{\sin (\sigma / \Delta w)}{\sigma / \Delta w}$,

and,

$G_{\Delta \sigma}(\sigma)=\exp \left(\frac{-\sigma^{2}}{2 \Delta \sigma^{2}}\right)$.

The two key parameters of this model are: the width, $\Delta w$, of $S_{\Delta w}(\sigma)$, which only depends on the resolution (see equation 6 ), and the line broadening which gives the width of $G_{\Delta \sigma}(\sigma)$. It is sometimes better to consider the broadening ratio $\Delta \sigma / \Delta w$ because it provides a measurement of the line broadening which is independent of the resolution.

An analytic formulation of this convolution is wellknown and is already implemented in the Herschel Interactive Processing Environment (Ott 2010). But we will show in the next section that its implementation is not optimal because of an overflow error that limits its use to large broadening ratios. We can start by giving this formulation as it is written in the Herschel Common Science System (HCSS) documentation ${ }^{2}$.

$S G(\sigma)_{\mathrm{erf}}=A e^{-b^{2}} \frac{\operatorname{erf}(a-i b)+\operatorname{erf}(a+i b)}{2 \operatorname{erf}(a)}$,

with

$a=\frac{\Delta \sigma}{\sqrt{2} \Delta w}$ and $b=\frac{\sigma-\sigma_{0}}{\sqrt{2} \Delta \sigma}$

where $A$ is the maximum value of the function, $\sigma_{0}$ its maximum position, $\Delta w$ is the width of the sinc and $\Delta \sigma$ the width of the Gaussian. This form will be considered as the basic erf formulation of the gaussian-sinc convolution (a demonstration of this result is given in Appendix A).

As mentioned above, if the line broadening is sufficiently small a pure sinc can be used as a reliable model. Then, at a given resolution there must exists a limit in signal-to-noise ratio (SNR) where the broadening is not measurable any more, i.e. when the difference, point by point, between a broadened line and a pure sinc becomes smaller than the noise standard deviation. This detectability limit is shown in Fig. 2 in terms of broadening ratio. The velocity disper-

2 http://herschel.esac.esa.int/hcss-doc-14.0

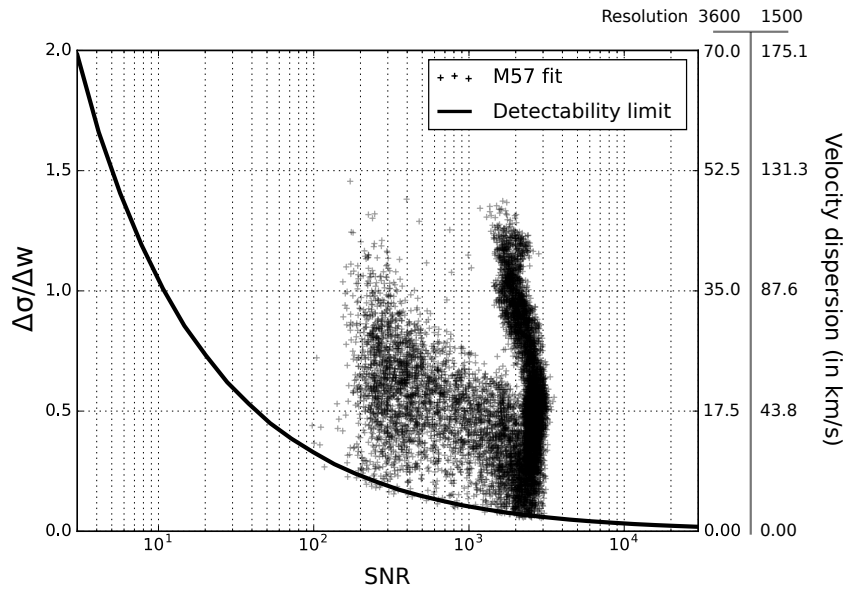

Figure 2. Minimum measurable broadening ratio $\Delta \sigma / \Delta w$ for a line with a given SNR. The corresponding velocity dispersion around $\mathrm{H} \alpha(656.28 \mathrm{~nm})$ at two different resolutions (3700 and $1500)$ has been reported on the figure. It scales linearly with the resolution. A randomly chosen set of points from the NGC 6720 cube fit is shown. The SNR of the data points is high because all the lines in the spectrum are fitted with the same broadening parameter.

sion $\Delta v$ corresponding to this broadening ratio has also been computed using the following equation:

$\Delta v=c \frac{\Delta \sigma}{\sigma}$

It clearly appears that, when the resolution decreases, the minimum broadening ratio needed to measure the same velocity dispersion becomes smaller. At a resolution of 1500 , a bubble expanding at a few $\mathrm{km} \mathrm{s}^{-1}$ will broaden a line with a SNR of 1000. Therefore, even at small resolution, we must be able to model an emission line with a small broadening ratio. In the next section we will show that the formulation given in equation 11 is not suitable for modelling small broadening ratios and we will propose a better formulation in this respect.

\subsection{A more computationaly robust analytic formulation}

Starting from equation 11, we can derive another formulation of $S G(\sigma)$ by using the definition of a Dawson integral $D(\sigma)$ :

$D(\sigma)=e^{-\sigma^{2}} \int_{0}^{\sigma} e^{y^{2}} d y=\frac{-i \sqrt{\pi}}{2} e^{-\sigma^{2}} \operatorname{erf}(i \sigma)$.

Replacing the error function by a Dawson integral everywhere in equation 11 gives what we will call the Dawson formulation of $S G(\sigma)$ :

$S G(\sigma)_{\text {Dawson }}=A \frac{D(i a+b) e^{2 i a b}+D(i a-b) e^{-2 i a b}}{2 D(i a)}$.

Overflow arises for both the error function and the Dawson integral when the imaginary part of the input becomes greater than 26.64 in 64-bit float representation (the limit 


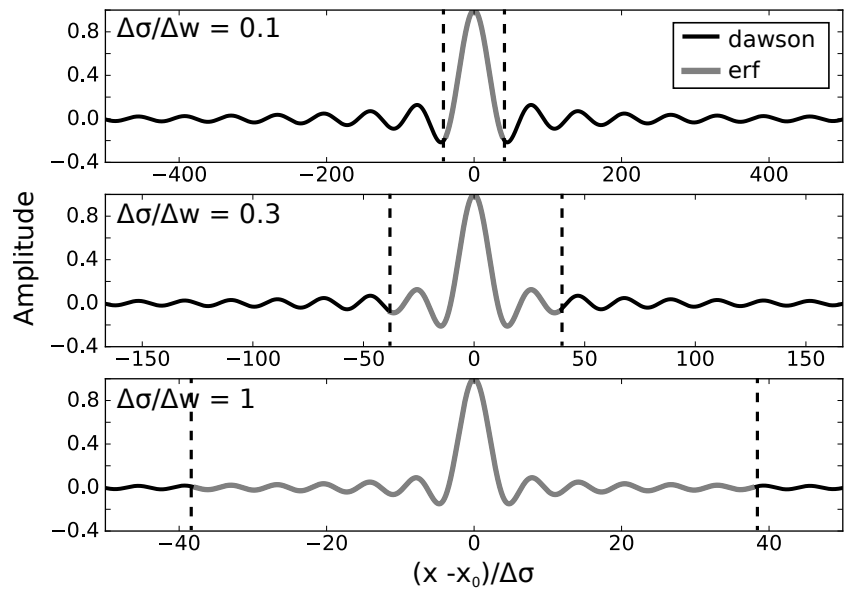

Figure 3. Comparison of Dawson and erf models for different values of $\Delta \sigma / \Delta w$. The truncation of the erf model due to overflow errors when $b>26.64$ (equivalent to $\left.\left(\sigma-\sigma_{0}\right) / \Delta \sigma>37.67\right)$ appears clearly.

is around 9.4 for 32-bit floats $)^{3}$. The erf model is thus limited by the value of $b$ which scales as $\left(\sigma-\sigma_{0}\right) / \Delta \sigma$. When $\Delta \sigma$ is small compared to the width of the sinc, the erf model cannot be evaluated after a few lobes (typically less than 1 if $\Delta \sigma<0.27 \Delta w$, see Fig. 3) where their relative amplitude is significantly high. When fitting a line, the truncated part can only be replaced with zeros as it is the case in the HCSS. It is possible to evaluate the relative error $\varepsilon$ as the ratio of the energy contained in the non-truncated part over the total energy of the line:

$\varepsilon=\frac{\int_{-\infty}^{+\infty} S G_{\text {erf }}(\sigma)^{2} \mathrm{~d} \sigma}{\int_{-\infty}^{+\infty} S G_{\text {Dawson }}(\sigma)^{2} \mathrm{~d} \sigma}$

We can see in Fig. 4 that this error can be greater than $70 \%$ for small values of $\Delta \sigma / \Delta w$ because when $(\Delta \sigma<0.2 \Delta w)$, even the central lobe cannot be fully evaluated (see Fig. 3 ).

Note that the Dawson model is also subject to an overflow error when $\Delta \sigma / \Delta w>37$. Such a high broadening level corresponds to an expansion velocity greater than $3500 \mathrm{~km} \mathrm{~s}^{-1}$ at a resolution of 1500 , which is easily observable in the AGN of numerous galaxies (Osterbrock \& Ferland 2006). It could be fitted with a simple Gaussian model but it's interesting to note that in this case the erf model can be used instead of the Dawson model.

\section{DESIGN OF A GAUSSIAN APODIZING FUNCTIONS FOR FOURIER TRANSFORM SPECTROSCOPY}

Apodization is frequently used to reduce the amplitude of the sidelobes of the ILS to obtain a more dispersive-like spectrum. The idea is to convolve the ILS with a function designed to have the desired properties. A convolution in the spectrum space is a product in the interferogram space and,

${ }^{3} \operatorname{erf}(26.64 i)>3.410^{306} i$ and $D(26.64 i)>1.410^{308} i$. The 64 -bit floating limit in python is around $1.710^{308}$.

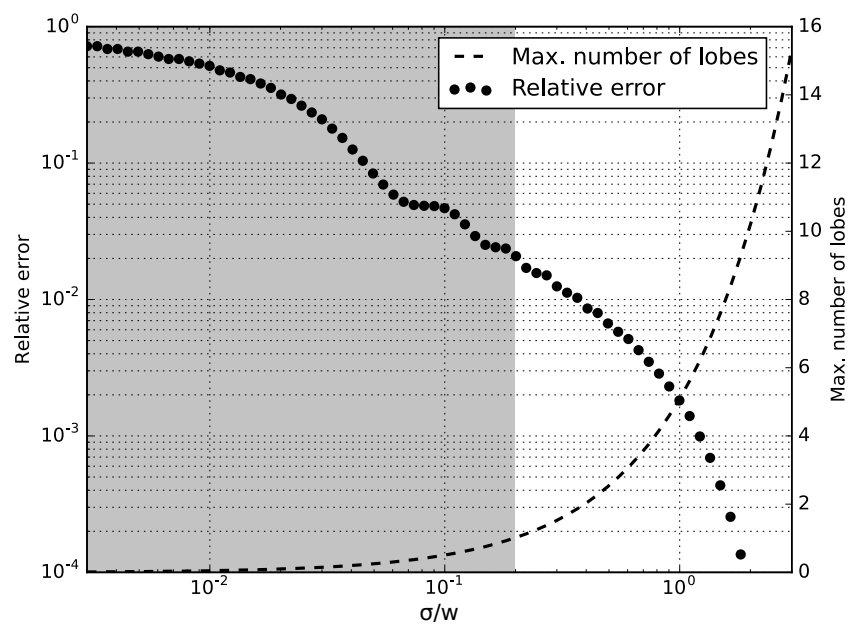

Figure 4. Limitations of the erf model. Due to overflow errors $S G_{\text {erf }}(\sigma)$ cannot be computed too far from the central lobe. The maximum number of lobes on one side of the function that can be represented and the relative error made because of the truncation of the model are presented (see text for details on the error evaluation). The region where even the central lobe is not fully evaluated is displayed in grey.

most of the time, this operation is better done on the interferogram before the Fourier transform (see, for example, Davis et al. 2001). $A(x)$ being the apodizing function and $I(x)$ the original interferogram, the apodization operation can be simply written as

$I_{A}(x)=I(x) A(x)$.

We have seen in the previous sections that the convolution of a sinc and a Gaussian will broaden the ILS and reduce the size of the sidelobes. Broadening the ILS will result in a reduced spectral resolution while the general shape of the instrumental line will become more Gaussian. If the ILS is well known (a sinc in the case of an ideal FTS) there is absolutely no need to apodize the spectrum. But, if, for some reasons, the ILS cannot be perfectly modeled, as it happens with non-ideal instruments, apodizing will smooth the ILS and help in reducing fitting artifacts coming from a bad model.

A lot of apodizing functions can be found in the literature (e.g. see Naylor \& Tahic 2007 and references therein), each one of them having its own particular use and properties. But when it comes to fitting, having the best model possible is of great importance. If we consider an ideal instrument with an ideal ILS, the ILS of an apodized spectrum can be described as being the result of the convolution of a sinc and the Fourier transform of the apodizing function. An analytic formulation of this ILS can be written as

$M(\sigma)=S_{\Delta w}(\sigma) * F T[A(x)](\sigma)$.

Now, if we want to analyze a Gaussian-shaped line of width $\Delta \sigma_{1}$ (see previous section) a more realistic model would be

$M(\sigma)=G_{\Delta \sigma_{1}}(\sigma) * S_{\Delta w}(\sigma) * F T[A(x)](\sigma)$.

Having an analytic formulation of such a fitting model can be difficult or even impossible to derive depending on the complexity of $F T[A(x)](\sigma)$. But if we consider using 
Gaussian apodizing functions of width $\Delta \sigma_{2}$ (and remembering that the Fourier transform of a Gaussian is also a Gaussian), the model of the apodized spectrum becomes

$$
\begin{aligned}
M(\sigma) & =G_{\Delta \sigma_{1}}(\sigma) * S(\sigma) * F T[G(x)](\sigma) \\
& =G_{\Delta \sigma_{1}}(\sigma) * S(\sigma) * G_{\Delta \sigma_{2}}(\sigma) \\
M(\sigma) & =G_{\Delta \sigma_{1 * 2}}(\sigma) * S(\sigma)
\end{aligned}
$$

where,

$\Delta \sigma_{1 * 2}=\sqrt{\Delta \sigma_{1}^{2}+\Delta \sigma_{2}^{2}}$.

Equation 22 comes directly from the fact that the convolution of two Gaussians is a Gaussian with a width given by equation 23 . The fitting model developed in the previous section can therefore also be used on Gaussian-apodized spectra. Even if Gaussian apodized functions may not have the best properties in terms of sidelobes magnitude reduction for a given broadening (Harris 1978) they are still interesting in this respect. Following the idea introduced by Naylor \& Tahic (2007), we have designed Gaussian apodizing functions with an adjustable broadening factor $b$, i.e. the factor by which the FWHM is increased when the spectrum is apodized. They can be described as simple Gaussian function,

$G(w, x)=\exp \left(-\frac{x^{2}}{\mathrm{MPD}^{2}} w(b)^{2}\right)$,

where,

$w(b)=b-1+2 \sqrt{2 \ln 2} \operatorname{erf}\left(\frac{\pi \sqrt{b-1}}{2}\right)$.

Because the resulting ILS of the spectra apodized with this set of functions can be perfectly modeled with the model derived in Section 2.3 (see equation 15) they have been implemented in ORBS, SITELLE's reduction pipeline (Martin et al. 2012), as the default apodizing function.

\section{NEW INSIGHTS ON THE VELOCITY STRUCTURE OF NGC 6720}

NGC 6720 (also known as M 57 or the Ring nebula) is one of the brightest planetary nebulae $(\mathrm{PNe})$ of the Galaxy. It has been extensively studied by numerous authors and techniques, making it an object of choice to evaluate the quality of the results obtained with the model depicted in the previous sections and try to provide new insights on its general structure. We refer especially to the most recent and extensive study of (O'Dell et al. 2013a, henceforth Od13) for a complete description of this object. As a general picture, this nebula is seen along its polar axis, so that its equatorial plane is nearly parallel to the plane of the sky. It consists of a dim core made of two lobes expanding along the polar axis and surrounded by a bright lower ionization region of higher density called the Main Ring which lies in the equatorial plane of the object. Both regions are optically thick and ionization bounded (Od13). All around the Main Ring we find the Inner Halo, recognizable at its petal-like structures which may be thin bubbles of ionized material (Guerrero et al. 1997, henceforth Gu97, and Od13). The Outer Halo is the farthest detectable structure. It presents a circular shape on the plane of the sky which suggests that it is a thin shell.
Table 1. Observation parameters of NGC 6720. Resolution is computed from the number of steps with respect to the ZPD position $(593-215=378)$ at the $\mathrm{H} \alpha$ wavelength and for an offaxis angle of $15.4^{\circ}$ that corresponds to the center of the field of view.

\begin{tabular}{cccccc}
\hline Filter & Order & $\begin{array}{c}\text { Step } \\
\text { size }(\mathrm{nm})\end{array}$ & $\begin{array}{c}\text { Step } \\
\text { number }\end{array}$ & $\begin{array}{c}\text { ZPD } \\
\text { position }\end{array}$ & $\begin{array}{c}\mathrm{R} @ \mathrm{OH} \alpha \\
\left(\theta=15.4^{\circ}\right)\end{array}$ \\
\hline SN3 & 8 & 2880.88 & 593 & 215 & 2650 \\
\hline
\end{tabular}

Both the Inner and the Outer Halo features are considered to be fossil radiation by Od13. The location of the different regions is shown in Fig. 5. If we examine the elliptical profile of the density shown in Fig. 5, the Main Ring corresponds to the region where the density is at its highest level compared to the Central Lobe and the Halo (see Fig.6).

\subsection{Observations with SITELLE}

NGC 6720 has been observed with SITELLE on August 8, 2015 during the commissioning of the instrument at CFHT (L. Drissen et al. 2016, in preparation, Baril et al. 2016). A data cube has been obtained in the SN3 filter $(647-685 \mathrm{~nm}$, including $\mathrm{H} \alpha$ as well as the [NII] and [SII] doublets) with a resolution $\mathrm{R}=2600$ and a seeing-limited spatial resolution of 0.8 " (see Table 1). This data has been fully reduced and calibrated with ORBS (Data Release 1, see T. Martin et al. 2016, in preparation and Martin et al. 2012). Flux calibration and transmission correction have been made from the observation of the spectrophometric standard planetary nebula M1-71 (Wright et al. 2005) obtained during the same observing run, as well as the observation of the spectrophotometric standard star GD71 (Bohlin et al. 2001) obtained in January 2016 through the same filter. A primary wavelength calibration has been done via the observation of a green He-Ne laser source at $543.5 \mathrm{~nm}$. This calibration has been refined by the measurement of the sky spectra, more specifically using the numerous Meinel $\mathrm{OH}$ bands present in the filter passband, around NGC 6720 and corrected for heliocentric velocity with ORCS (Martin et al. 2016, in preparation). The pixel-to-pixel calibration error on the velocity is estimated to be below $0.5 \mathrm{~km} \mathrm{~s}^{-1}$. The absolute velocity calibration is estimated to be around $1 \mathrm{~km} \mathrm{~s}^{-1}$ (see appendix B for details).

With an integration time of 2.5 hours, the SNR of the spectra covering most of the nebula is high enough to study the dynamics with some precision: individual spectra in the brightest regions exhibit lines of $\mathrm{H} \alpha$ and $[\mathrm{N} \mathrm{II}] \lambda 6583$ with a SNR greater than 100 . One of the approximately 280000 spectra, taken near the center of nebula (where the velocity dispersion is the highest), is shown in Fig. 7.

\subsection{Evaluation of the fitting model}

To illustrate the importance of using a model based on the convolution of a sinc and a Gaussian we show in Fig. 7 the results of two fits made on this spectrum. One of the fits makes use of the line model developed in the previous section and the other is based on a pure sinc. The residual near the first lobes of the emission line is more than ten times higher when using a pure sinc model and exhibits a 

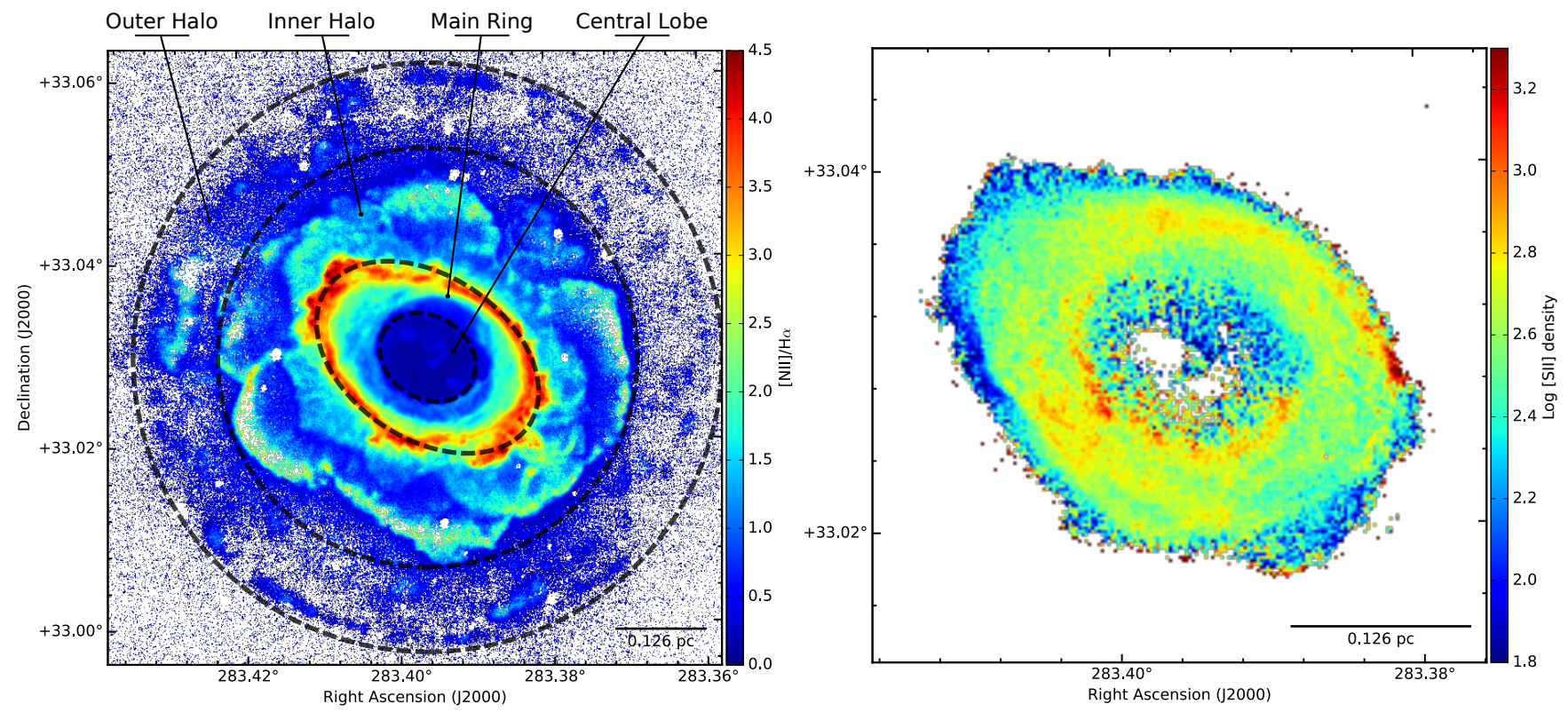

Figure 5. Left: Map of the $[\mathrm{N} \mathrm{II}] \lambda 6584$ / $\mathrm{H} \alpha$ line ratio of NGC 6720. The approximate contours of the different regions of the nebula are indicated. Right: Map of the [S II] density of the central part of NGC 6720. Following O'Dell et al. (2013b), this map has been computed with the relation $\log n_{e}=4.705-1.9875 S(671.6) / S(673.1)$ from Osterbrock \& Ferland (2006) which is accurate for densities in the range $100 \mathrm{~cm}^{-3}-3000 \mathrm{~cm}^{-3}$. The uncertainty on the estimate of the $[\mathrm{N} \mathrm{II}] / \mathrm{H} \alpha$ ratio and the density is shown in Fig. D1.

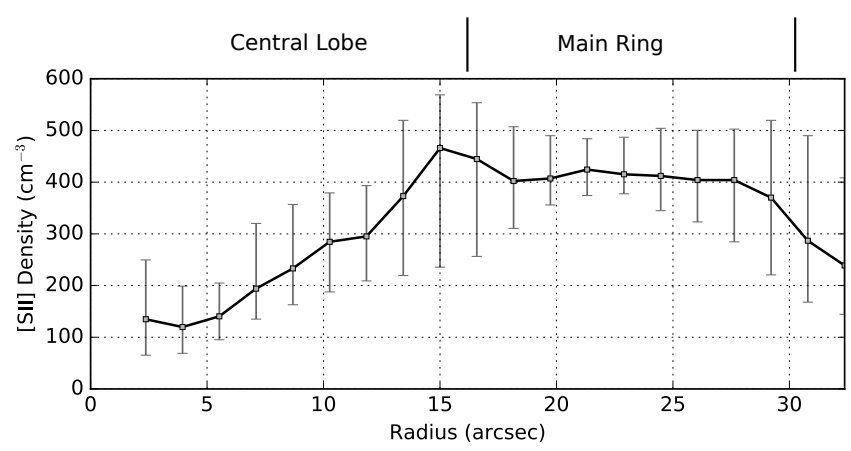

Figure 6. Elliptical profile of the $[\mathrm{S} I \mathrm{I}]$ density of the central part of NGC 6720 considering a mean temperature of $10000 \mathrm{~K}$. The density map has been convoluted with a $3 \times 3$ Gaussian kernel. Density has been computed from the [S II] ratio with Pyneb (Luridiana et al. 2012), the Python implementation of the nebular package (Shaw \& Dufour 1995). The radius is given for the minor axis of the ellipses. The parameters of the ellipse scaled to obtain this profile is the Main Ring ellipse shown on Fig. 5. The position of the Central Lobe ellipse and the Main Ring ellipse along the minor axis are indicated. The error bars show the 16th and 84th percentiles of the distribution along the ellipses.

typical modelling error. The implementation of the Dawson model in ORCS, the fitting engine developed for SITELLE's data (Martin et al. 2015), is robust enough to be used on a regular basis to fit entire $3 \mathrm{D}$ cubes. With this software we have mapped the velocity dispersion of the ionized gas in NGC 6720. Because the angular size of a pixel is 0.32 ", which is more than two times smaller than the average seeing of the observation, the spectral cube has been binned
$2 \times 2$ to enhance the SNR without losing too much spatial resolution.

As it is shown in Fig. 8 and Fig. D2, the velocity dispersion along the line of sight has been measured on all the spectra covering the object with an uncertainty as low as a few $\mathrm{km} \mathrm{s}^{-1}$. Modelling the spectra over the entire spectral range covered by the filter is key for this fitting procedure because the secondary lobes of individual lines are interfering with each other up to the filter edges. It is thus important to model a line with all its lobes even for small broadening ratios. In the case of NGC 6720 , the emission lines with a velocity dispersion around $10 \mathrm{~km} \mathrm{~s}^{-1}$ have a broadening ratio of 0.2. With the erf implementation even the first lobe could not have been computed (see Fig. 3).

ORCS uses a least-square Levenberg-Marquardt minimization algorithm (Levenberg 1944; Marquardt 1963) to fit the data. All the lines are fitted simultaneously and some parameters are grouped together to enhance the quality of the fit. The velocity and the broadening are considered to be the same for the $[\mathrm{NII}]$ and $[\mathrm{SII}]$ lines (which reduces the number of velocity parameters from 4 to 1 ). $\mathrm{H} \alpha$ velocity and broadening are fitted independently from the other lines. The Levenberg-Marquardt algorithm assumes the Gaussianity of the noise distribution and the fact that the contours of the objective function are "nearly elliptical in the immediate vicinity of the minimum" (Marquardt 1963). It is important to test the validity of this hypothesis if we want to make sure that the estimation on the parameters is unbiased and that the calculated uncertainty (derived from the covariance matrix) is correctly estimated. We have used a Monte-Carlo-Markov-Chain algorithm implemented in the emcee Python library (Foreman-Mackey et al. 2012) to get the marginalized distribution of the posterior probability of each parameter (see Fig. C1). We find that the ampli- 


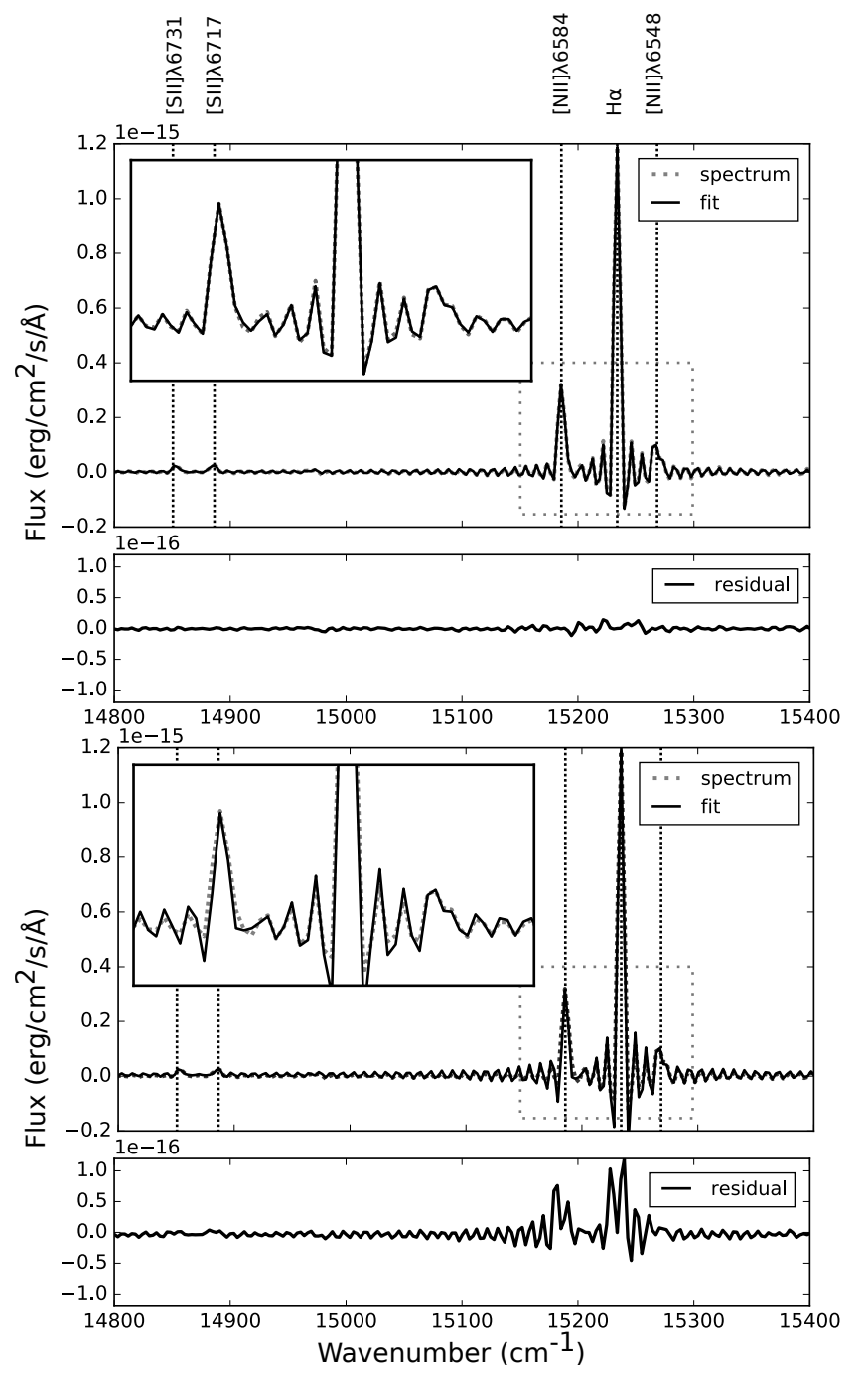

Figure 7. Top: Example of a fit on one NGC 6720 spectrum realized with the $S G_{\text {Dawson }}(\sigma)$ model developed in this article. The SNR of the lines ranges from 600 , for $\mathrm{H} \alpha$, to 20 for the [SII] lines. The fit, superimposed on the original data, and the residuals, are shown. A zoom on the $\mathrm{H} \alpha$ region illustrates the quality of the model used. Bottom: Fit of the same spectrum as on the top panel but realized with a pure sinc model. The high residual near the first lobes of the lines indicates a poorly fitted model.

tude and velocity parameters show a circular 2D distribution around the best value and a nearly Gaussian marginalized distribution. When the value of the broadening parameter is sufficiently high (e.g. the [N II], [S II] broadening which is around $12 \mathrm{~km} \mathrm{~s}^{-1}$ in the example on the figure) the distribution is nearly ideal. But it shows an asymmetric distribution when it is approaching zero (which is the case of the $\mathrm{H} \alpha$ line). It means that very low broadening values (under a few $\mathrm{km} \mathrm{s}^{-1}$ down to zero) can be systematically biased to slightly higher values.

\subsection{Study of the velocity dispersion in NGC 6720}

\subsubsection{Limitations}

Before we start any analysis of the broadening and velocity maps obtained with SITELLE (see Fig. 8 and Fig. D2), we must consider some inherent limitations to the use of low-resolution data. At a resolution of 2600 , in the case of a PNe like NGC 6720, line broadening has 3 main physical origins, all of the same order of magnitude: shell expansion (a few $\left.10 \mathrm{~km} \mathrm{~s}^{-1}\right)$, thermal broadening ${ }^{4}\left(9.1 \mathrm{~km} \mathrm{~s}^{-1}\right.$ for $\mathrm{H} \alpha$ and $2.4 \mathrm{~km} \mathrm{~s}^{-1}$ for $[\mathrm{N} \mathrm{II}] \lambda 6584$ at a temperature of $10000 \mathrm{~K}$ ) and possibly turbulence (also around $10 \mathrm{~km} \mathrm{~s}^{-1}$ see O'Dell et al. 2013b; O'dell 1991; Medina et al. 2014 and references therein). Another source of line broadening is related to the instrument and comes from the modulation efficiency loss at large OPD. This problem is well documented in Baril et al. (2016) and might account for the larger measured velocity expansion of the shells (see section 4.3.3). The commissioning data has been taken without tilt correction so that we can expect a modulation efficiency loss of up to $25 \%$ at the MPD (that corresponds to 700 laser fringes of $1550 \mathrm{~nm}$ ). We have simulated the effect of this loss on line broadening and estimated that it may add at most $10 \mathrm{~km} \mathrm{~s}^{-1}$ to the global broadening.

We have estimated the amount of broadening added by the turbulent motion and complex internal motion by comparing the expansion velocity derived from our data (see section 4.3.3) to the one measured by other authors and found that it lies between 10 and $15 \mathrm{~km} \mathrm{~s}^{-1}$ for [N II] and between 20 and $25 \mathrm{~km} \mathrm{~s}^{-1}$ for $\mathrm{H} \alpha$ (after subtraction of the thermal broadening and the instrumental broadening). Note that turbulent motion cannot be separated from other complex internal motions which would provide more than the simple two emission lines we would expect for an ideal expanding shell. The observation of more than two emission lines is reported in several high-resolution studies (Gu97; Od13). Therefore, despite the small uncertainty of our measurement, the derived value of the expansion velocity is an upper limit that contains some unknown added quantity which can vary from one pixel to another.

We know that the line broadening is mainly caused by the expansion of a thin shell, at least in the core and the Main Ring regions. So that at a high resolution we should see two lines instead of one separated by twice the expansion velocity. At low resolution the resulting line can be treated as a broadened Gaussian line but this model is only an approximation. First of all, we have checked that this is a good approximation only if the line separation is less than half the FWHM when the SNR is smaller than $1000\left(\right.$ e.g $60 \mathrm{~km} \mathrm{~s}^{-1}$ at a resolution of 2600). In this case the differences between the model and the real spectrum are smaller than the noise (assuming a Gaussian noise). Secondly, when the two emission lines do not have the same intensity we can suspect that the centroid of the low-resolution line will be shifted, causing a bias on the estimation of the line velocity. There must also be some reduction of the measured broadening. Fig. 9 shows the resulting low-resolution line when the relative amplitude

4 The broadening values are expressed in terms of Gaussian width. In terms of FWHM we have $21.5 \mathrm{~km} \mathrm{~s}^{-1}$ for $\mathrm{H} \alpha$ and $5.7 \mathrm{~km} \mathrm{~s}^{-1}$ for $[\mathrm{N} \mathrm{II}] \lambda 6584$ at a temperature of $10000 \mathrm{~K}$. 

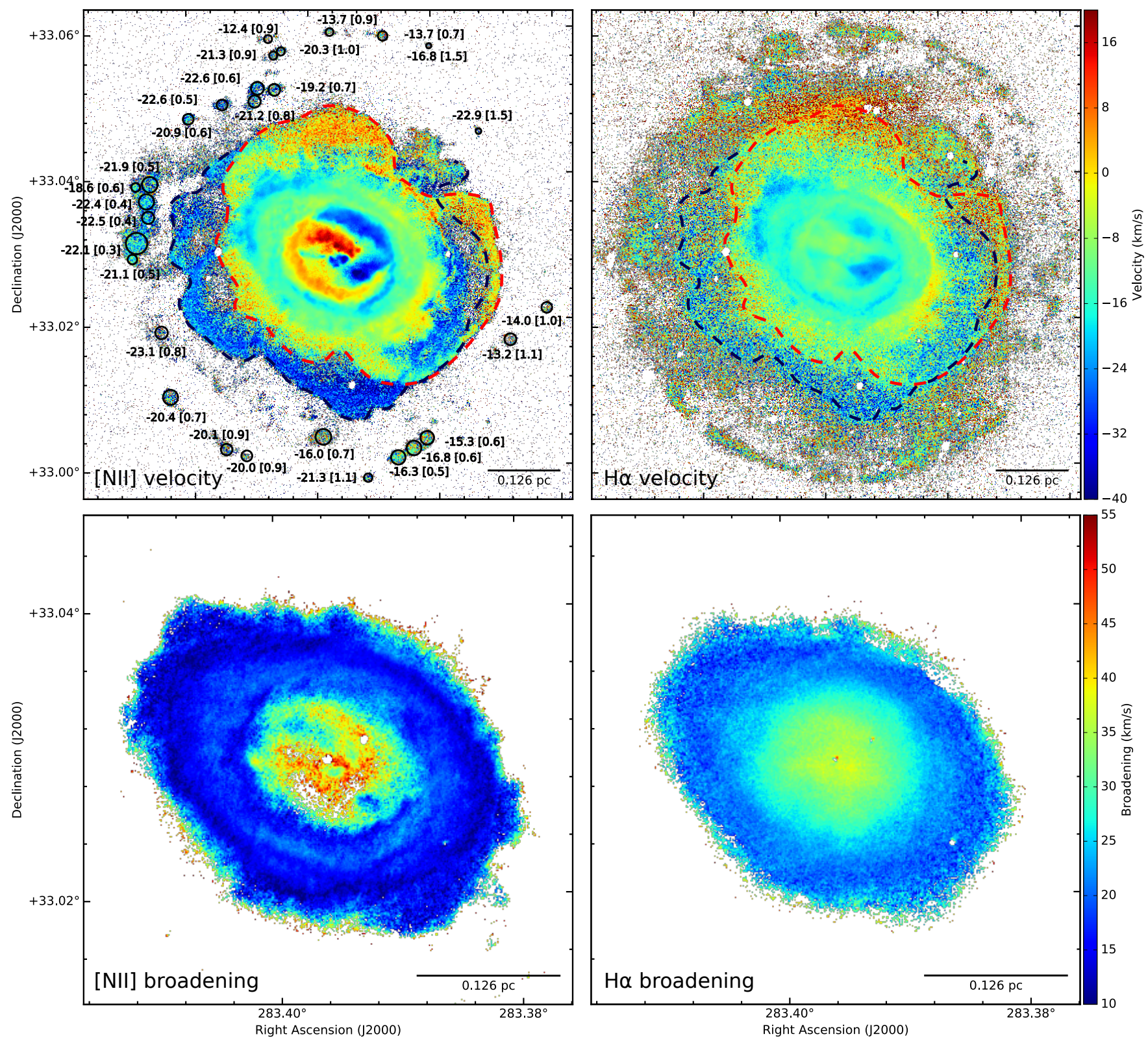

Figure 8. Velocity (top) and broadening (bottom) maps of [N $\mathrm{NI}] \lambda 6584$ and $\left[\mathrm{N}_{\mathrm{II}}\right] \lambda_{6548}$ (left) and H $\alpha$ (right). The two [N II] lines have been fitted together with the same velocity and broadening parameters to enhance the precision of the fit. Color scales are indicated on the right of the figure. Maps of the uncertainties on these estimates of the velocity and the broadening are shown in Fig. D2. Top-left: The velocity of the ionized features at the boundary of the Outer Halo has been computed from the integrated spectra. The aperture used for each region is indicated by a black circle along with the estimate of the velocity and its uncertainty. The approximate boundaries of the Halo bipolar structures moving away and towards us are indicated with dotted lines respectively in red and blue. Those boundaries are reported in the $\mathrm{H} \alpha$ velocity map (top-right panel).

of the second line changes. The expected line shift appears clearly. We can also have a look at the bottom of Fig. 9 to see how the measured velocity and broadening are affected. We can notice that the velocity shift scales nearly linearly with the amplitude ratio. But the broadening is much less affected. In fact it starts to be underestimated by more than $10 \%$ only when the amplitude of the second line is $40 \%$ the amplitude of the first line. An empirical fit to this data gives an estimation of the relative error $\Delta \sigma_{\text {Error }}$ for a given amplitude ratio $r$

$\Delta \sigma_{\text {Error }} \simeq 1-\operatorname{erf}\left(2 r^{0.6}\right)$.

We can therefore conclude that, at low resolution, the estimation of the expansion velocity of a shell via the broadening of an emission line will give reliable results while the measure of the velocity itself, even at high SNR, is subject to a shift equal to half the broadening. If we look at the core region of the $[\mathrm{N}$ II] velocity map in Fig. 8, we can see a very large velocity difference (around $30 \mathrm{~km} \mathrm{~s}^{-1}$ ) which is much 

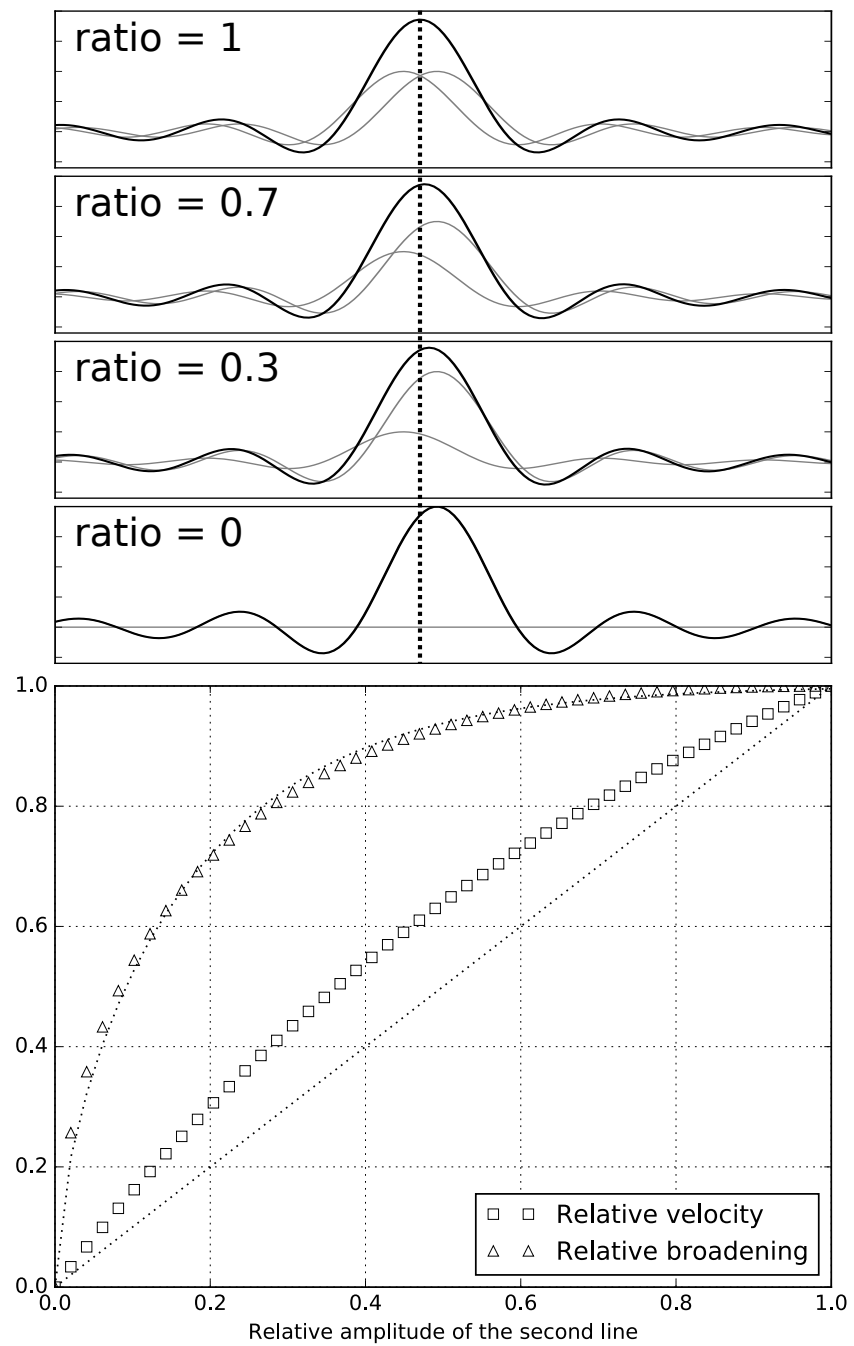

Figure 9. Top: Sum of two unresolved $\mathrm{H} \alpha$ lines observed at a resolution of 2600 created by an very thin shell expanding at a velocity of $40 \mathrm{~km} \mathrm{~s}^{-1}$ for different intensity ratios of the lines. The real center of the two lines is indicated with a dotted line. Bottom: Estimated velocity and broadening for different ratios of the intensity of two lines emitted by a thin shell expanding at a velocity of $40 \mathrm{~km} \mathrm{~s}^{-1}$ observed at a resolution of 2600 . An empirical fit of the broadening variation (see text for details) and the one-to-one line are indicated with dotted lines.

smaller in the $\mathrm{H} \alpha$ map. In fact the front and the back of the nebula do not have the same intensity along the line of sight and the centroid of the line is simply shifted towards the most luminous portion of the shell. We are thus not looking at a very disturbed velocity field at the center of NGC 6720 . What the velocity map tells us here is probably that the ionized material on the central shell is not homogeneously distributed. Moreover the apparent high symmetry of the velocity field suggests that the ejected material has been distributed with a strong central symmetry with respect to the AGB star. The extracted velocity maps must therefore be understood as a lower limit in the case of an expanding shell where two lines are possibly present, but unresolved, along the line of sight.

\subsection{2 $3 D$ velocity map of the Main Ring and the Central Lobes}

Given the limitations explained in the previous section, it appears somehow naive to derive a $3 \mathrm{~d}$ model of the $[\mathrm{N}$ II] shell from our low resolution data. But even if the resolution is lower than that of echelle spectra, we must highlight the fact that it is the first time velocity data is obtained with an homogeneous spatial sampling over the entire nebula. It is interesting to compare what we can derive from our data to the most complete and precise study of NGC 6720 from O'Dell et al. (2007) (henceforth Od07) based on longslit spectroscopy at different position angles centered on NGC 6720 . The high spectral resolution $(R \sim 42000)$ of their data is perfect to clearly separate the lines emitted from both sides of the shell. But the spatial coverage is inhomogeneous and the data must be interpolated between one position angle and the next, leading to an increasing error as one moves away from the center of the slits.

We have constructed a $3 \mathrm{D}$ map of the velocity dispersion based on our data at 3 different ranges of flux (high, medium and low surface brightness) in order to mimic the figures shown in Od07. They are presented in Fig. 10. Even if the models are not probing the same parameters, they appear very similar in many ways. The major difference can be seen at the boundary of the region (especially on the lower intensity one) where the line broadening, after a general decrease with an increasing radius, starts to increase again at the boundary of the region. This general trend also appears clearly in Fig. 11 and is reported in Gu97. The increasing broadening on the edges of the main ring of the nebula gives a less spherical shape to our model when compared to the model of Od07. The region affected corresponds to the inner halo of the nebula, where the density decreases (see Fig. 6 and Fig. 11 of Od07). The increasing broadening in this region comes from a more complex velocity field due to the superposition of the main ring shell and the halo shell. According to Gu97 the inner halo is the envelope from the remnant red giant wind which has a continuous density distribution, creating a broad component instead of two emission lines like the main nebula shell. The analysis of Od13 favors the hypothesis of an expanding shell in the halo. This is also what suggests our data (see Section 4.4). If we look at the Central Lobe, the general shape of the front surface is similar but it appears exaggerated in our model. This certainly comes from the shift of the centroid of the line that comes from the unequal surface brightness of the two expanding fronts of the shell along the line of sight. This effect is explained with more details in Section 4.3.1.

\subsubsection{Elliptical profile}

The inner region of the nebula encompasses a Central Lobe where the emission is generally low and the $[\mathrm{N} \mathrm{II}] / \mathrm{H} \alpha$ ratio is smaller than 0.5 and the Main Ring, the brightest part of the nebula where the $[\mathrm{N} \mathrm{II}] / \mathrm{H} \alpha$ ratio is greater than 1 and goes up to 4 at its boundary (see Fig. 5 and Od13). We have demonstrated in Section 4.3.1 that the broadening was not too much affected by the intensity ratio between the two lines of an expanding shell. Therefore, its value gives a reliable estimation of the radial velocity difference between these lines. Given the fact that the nebula is seen nearly 

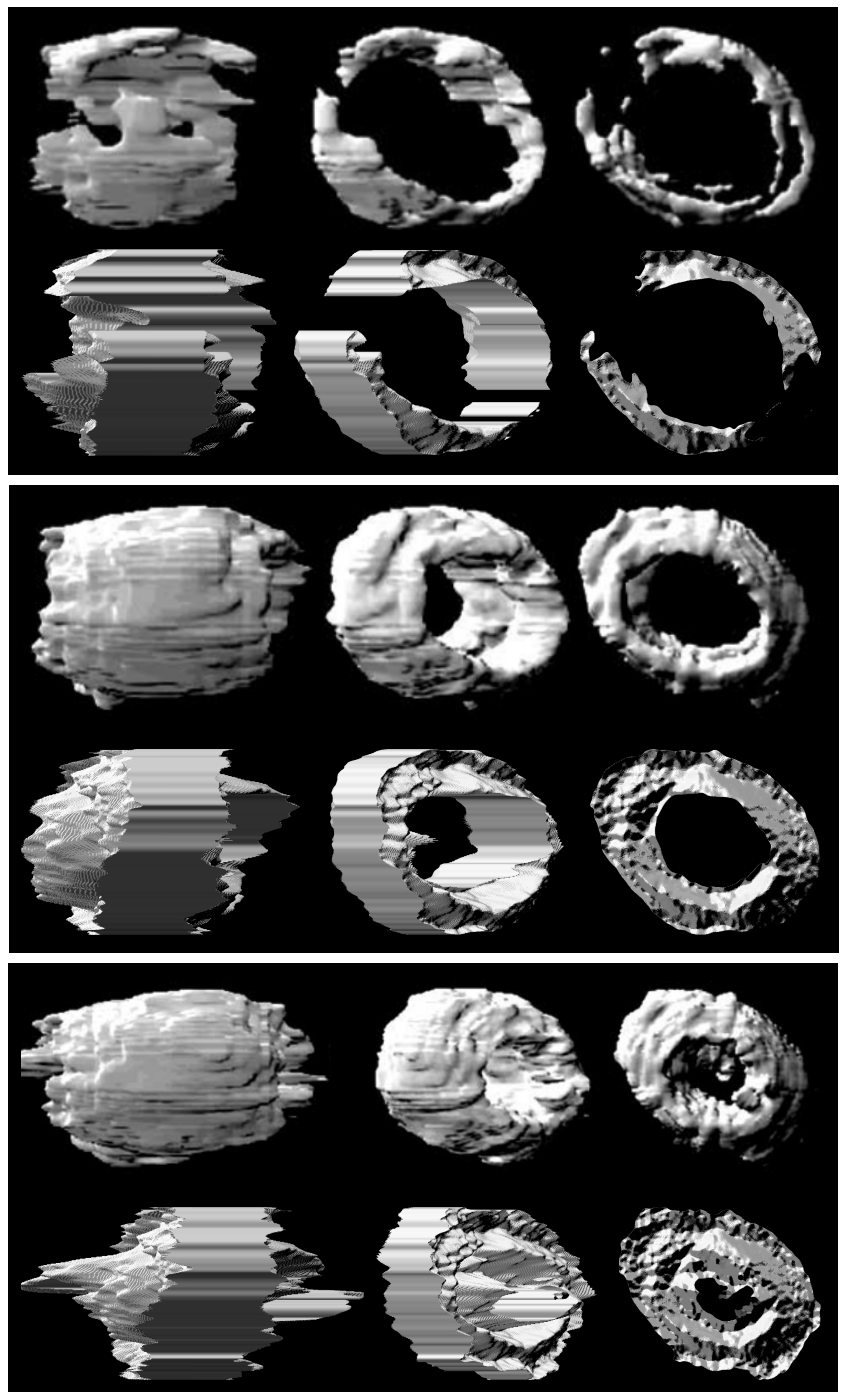

Figure 10. Opaque reconstruction determined from SITELLE's low resolution data (below) compared with Od07 (above) for 3 different flux levels (high, medium and low flux from top to bottom). Volumes are shown from three different directions: $90^{\circ}, 30^{\circ}$ and along the line of sight from left to right. North is up and east to the left.

pole-on and presents a good cylindrical symmetry along the polar axis, it is possible to compute the elliptical profile of the broadening of $[\mathrm{N}$ II] and $\mathrm{H} \alpha$. Those profiles are shown in Fig. 11. The high effective temperature of the central star creates a thin ionized front that is very well traced by the $[\mathrm{N} \mathrm{II}]$ lines. If we look at the broadening profile of the [N II] line, the elliptical distribution of the radial velocity in the Central Lobe appears clearly. The higher than expected standard deviation of its value along each radius with respect to the small scatter of the median values along the fitted elliptical profile is intriguing. This is most certainly due for a good part to the effect of the large difference of intensity of the two lines along the line of sight in this region (see Section 4.3.1). Even if the broadening value is less affected, we can see that it is not negligible. The small scatter of the median value along the fitted elliptical profile suggests that the expansion velocity of the ionized region presents
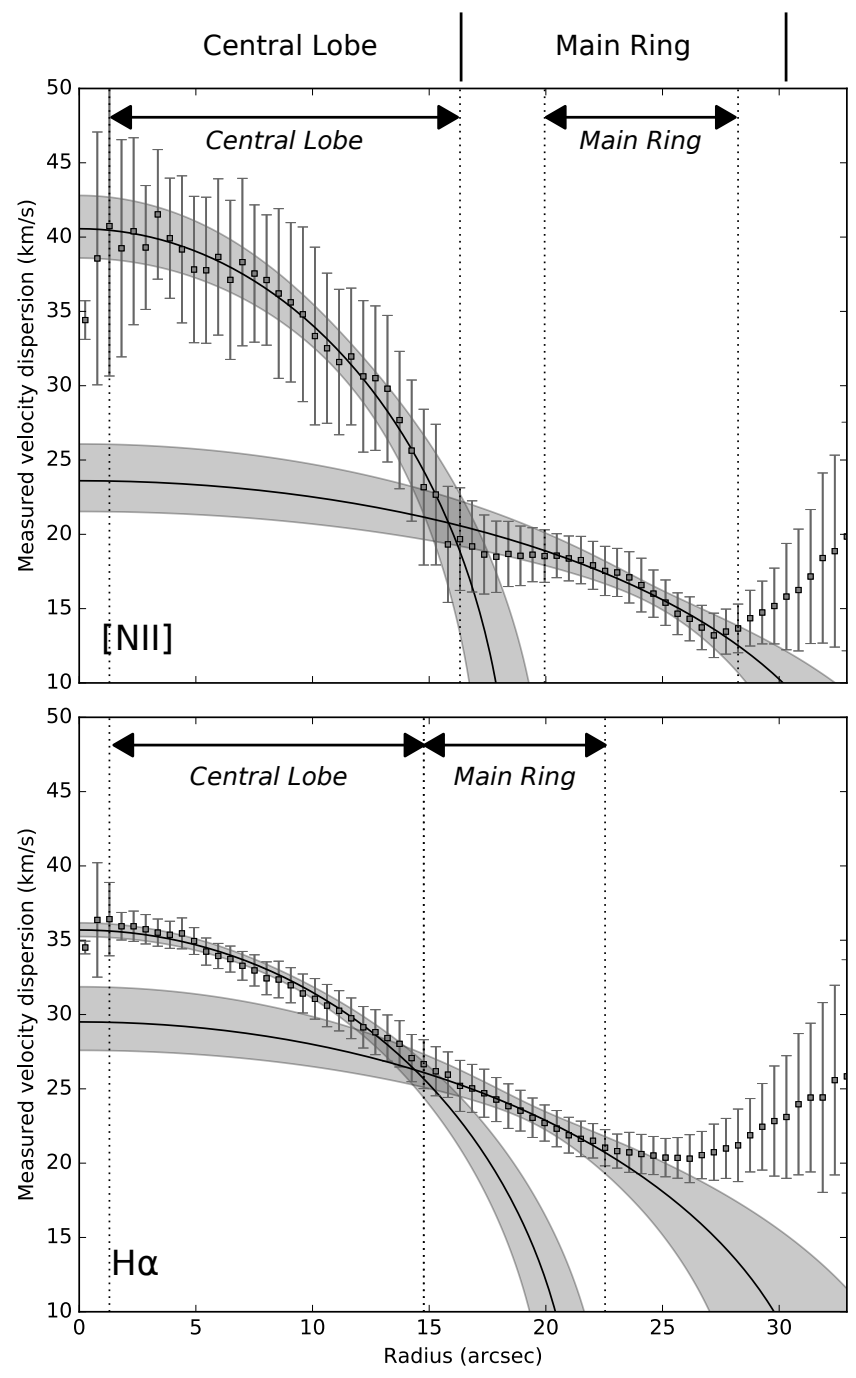

Figure 11. Elliptical profiles of the broadening of the [N II] lines (top) and the $\mathrm{H} \alpha$ line (bottom). The radius given is measured along the minor axis of the ellipses. The scale ellipse used to construct this figure corresponds to the Main Ring ellipse as shown in Fig. 5. For each profile, two elliptical expansion models have been fit (shown in solid black) with a Monte-Carlo Markov-Chain algorithm (see text for details). The error bars show the standard deviation of the broadening distribution along the ellipses. The regions used to fit the expansion models of the Central Lobe and the Main Ring are indicated with dotted lines. The regions defined by the models comprised between the 16th and 84th percentiles for each fit are shown in grey.

a very good central symmetry with respect to the ionizing star, which therefore indicates a very good central symmetry of the physical properties (temperature, density and abundances) of the medium crossed by the ionization front since its origins. The same central symmetry must have then be respected by the oldest $\mathrm{AGB}$ winds which remnants constitute the ionized material of the Outer Halo (see Section 4.4). Also, the presence of two shells expanding at different velocities, already deduced by Od13, can be confirmed. The faster shell corresponds to the central core of the nebula where the density is smaller while the slower one corresponds to the main ring where the density is much higher (see Fig. 5). 
An elliptical model has been fit to the data to compute the expansion velocity of each shell. If we consider the expansion velocity to be the same in all directions then a pure ellipsis is a perfect model. But we have to add to this model a constant velocity component which comes from thermal broadening, turbulence and the dispersion of the expansion velocity around its mean value.

$\sigma=\sqrt{\sigma_{\mathrm{T}}^{2}+\sigma_{\mathrm{V}}^{2}+V_{\mathrm{Exp}}^{2}\left(1-\frac{r}{r_{0}}\right)^{2}}$,

where $\sigma$ is the measured broadening, $V_{\mathrm{Exp}}$, the expansion velocity, $\sigma_{\mathrm{T}}$, the thermal broadening, $\sigma_{\mathrm{V}}$, the dispersion of the velocity coming from other origins, $r$ is the radius from the center and $r_{0}$ the radial scale parameter. We have tried to fit such a model with a Markov Chain Monte-Carlo method but the lack of data at the very edges of the shell, where the relative importance of the other sources of broadening becomes significant, does not permit to fit a model with so many parameters. Occam's razor clearly gives the preeminence to the simplest model:

$\sigma=\sqrt{V_{\operatorname{Exp}}^{2}\left(1-\frac{r}{r_{0}}\right)^{2}+\sigma_{\mathrm{T}}^{2}}$,

With this method, the fitted expansion velocity is $40.5 \pm 2.2 \mathrm{~km} \mathrm{~s}^{-1}$ for the central lobe and $23.5 \pm 2.2 \mathrm{~km} \mathrm{~s}^{-1}$ for the main ring which are both a few $\mathrm{km} \mathrm{s}^{-1}$ above the expansion velocities measured by Od13 (the given velocities are corrected for the estimation of the thermal broadening of $[\mathrm{N} \mathrm{II}]$ at $10000 \mathrm{~K})$. Note that only the outer part of the main ring has been used for the fit as the inner portion does not follow an elliptical shape. The expansion velocity of the PDR follows very well the density profile (see Fig. 5) suggesting that the ionization front is moving faster in low density regions.

If we look at the fit made for the $\mathrm{H} \alpha$ broadening, a simple elliptical model fails to explain its really homogeneous disk-like shape. This most certainly reflects the fact that hydrogen is ionized over a much deeper layer than $[\mathrm{NII}]$, thus smoothing the velocity differences between the Main Ring and the Central Lobe. The sum of two elliptical shells better explains the overall profile of the $\mathrm{H} \alpha$ broadening. The fitted expansion velocity in the core, corrected for the estimation of the thermal broadening of $\mathrm{H} \alpha$ at $10000 \mathrm{~K}$, is $34.5 \pm 0.5 \mathrm{~km} \mathrm{~s}^{-1}$. The fitted expansion velocity of the outer shell that corresponds roughly to the Main Ring is $28.1 \pm 2.2 \mathrm{~km} \mathrm{~s}^{-1}$.

\subsection{Study of the Halo}

As Od13 suggested, "the radial velocity of the Halo features are difficult to determine because of their low surface brightness". Up to now, the only data available with enough signalto-noise ratio comes from the long slit study of Gu97. Their Fig. 8 shows a broad component that narrows from the Inner Halo to the edge of the Outer Halo. Od13 see in this figure a velocity ellipse corresponding to an expansion velocity of $15 \mathrm{~km} \mathrm{~s}^{-1}$. Despite the low resolution of our spectra, its high spatial resolution combined with an integration time of 2.5 hours give us the deepest and most complete picture of the Halo up to date. A general examination of the [N II] velocity map in Fig. 8 shows that the Inner Halo is composed of two ionized fronts with a velocity difference of more than $30 \mathrm{~km} \mathrm{~s}^{-1}$. These two fronts are obvious when we look out of the Main Ring but near the Main Ring, the dim petals of the Inner Halo are likely to be hidden by its very high surface brightness. The two fronts are similar in shape but they appear shifted with respect to one another along the minor axis of the nebula: one front is expanding towards us in the SW region while the other is expanding away from us in the NE direction. According to Od13, the nebula is supposed to be tilted by $5^{\circ}$ along the minor axis which creates a small velocity difference of $5 \mathrm{~km} \mathrm{~s}^{-1}$. The velocity difference between the two fronts thus cannot be the result of this tilt. Moreover, the front expanding away from us is also visible in the SW region. This suggests that the Inner Halo is a shell expanding in an inhomogeneous medium with a strong bipolar symmetry. The two poles of the ionized front are at least roughly aligned with the polar axis of central region of the nebula. The expansion velocity must be at least of $15 \mathrm{~km} \mathrm{~s}^{-1}$ but this is a lower limit because the expansion velocity cannot be measured along the line of sight. The relative velocity of the whole shell with respect to the Local Standard of Rest is around $-15 \mathrm{~km} \mathrm{~s}^{-1}$. In the regions where the two fronts are overlapping the measured velocity is also around this value. This effect which comes from the mix of two emission lines with a similar intensity is explained in details in Section 4.3.1. The arcuate emission features are coming from the complex morphology of the ionized front composed of portions of bubbles.

The small ionized regions lying on the edge of the Outer Halo are too dim to be studied with a good spatial resolution. We have thus used integrated spectra of small circular regions to obtain a more precise measurement of their velocity (see Fig. 8). Interestingly, most of the Outer Halo features have an LSR velocity around $-20 \mathrm{~km} \mathrm{~s}^{-1}$, that is very near the mean velocity of the Inner Halo bipolar shell. This suggests that the Inner Halo and the Outer Halo may be in fact the same shell. This shell would be made of a two bright poles. The dim and inhomogeneous emission regions distributed along a circle can be seen because of a projection effect at the boundaries of the Halo shell.

The overall symmetry of the ejection of material in the core and the Halo is remarkable. The velocity map suggests that the SW part of the core is filled with material moving away from us while the SW part of the Halo is filled with material moving away from us. The exact counterpart of these opposite ejections in terms of volume and shape can be seen in the NE part of the core and the Halo.

\section{CONCLUSIONS}

We have defined a better implementation of a sinc instrumental line convolved with a Gaussian profile and have designed the corresponding set of Gaussian apodizing functions that are now used by the reduction pipeline ORBS. We have used this model for the analysis of the commissioning data obtained with SITELLE at CFHT that covers the planetary nebula NGC 6720. Our major conclusions are listed below.

(i) Our implementation of the convolution of a sinc and a Gaussian profile is much better at fitting small broadening values. 
(ii) A complete spectrum model consisting of multiple lines and a low-order continuum can be fitted to the spectra of NGC 6720 and respects the underlying hypotheses of the Levenberg-Marquardt minimization algorithm.

(iii) We have demonstrated that the broadening of an unresolved line emitted by a shell could give a robust estimation of its expansion velocity while the position of its centroid was greatly affected by the intensity ratio of the two lines emitted by both fronts of the shell along the line of sight.

(iv) We have derived the first complete density map of the central part of the nebula which clearly shows that the Main Ring is the region of highest density.

(v) The study of the broadening of the [N II] lines have clearly demonstrated, as Od13 originally suggested, that the Main Ring and the Central Lobe are two different shells with different expansion velocities.

(vi) We have derived the deepest and the most spatially resolved velocity maps of the Halo in $[\mathrm{N} \mathrm{II}]$ and $\mathrm{H} \alpha$.

(vii) The study of the velocity of the Halo features reveals that the brightest bubbles are originating from two bipolar structures with a velocity difference of more than $35 \mathrm{~km} \mathrm{~s}^{-1}$ lying at the poles of a possibly unique Halo shell expanding at a velocity of more than $15 \mathrm{~km} \mathrm{~s}^{-1}$.

The great quality and the quantity of data obtained with SITELLE on this object reveals much more on this nebula than the few aspects covered here. The investigation of these aspects will be published in a next article. The observation with SITELLE of the lines [O III] $\lambda \lambda 4959,5007$, [O III] $\lambda 4363$, the $[\mathrm{O}$ II $] \lambda 3727$ doublet and the $[\mathrm{N}$ II $] \lambda 5755$ line will certainly permit a much deeper and more precise study of this object.

\section{ACKNOWLEDGEMENTS}

Based on observations obtained with SITELLE, a joint project of Université Laval, ABB, Université de Montréal and the Canada-France-Hawaii Telescope (CFHT) which is operated by the National Research Council (NRC) of Canada, the Institut National des Science de l'Univers of the Centre National de la Recherche Scientifique (CNRS) of France, and the University of Hawaii. LD is grateful to the Natural Sciences and Engineering Research Council of Canada, the Fonds de Recherche du Québec, and the Canadian Foundation for Innovation for funding.

\section{REFERENCES}

Baril M. R., et al., 2016, in Evans C. J., Simard L., Takami H., eds, Proceedings of SPIE. International Society for Optics and Photonics, p. 990829, doi:10.1117/12.2232075

Bernier A.-P., 2006, in Proceedings of SPIE. SPIE, pp 626949 626949-9, doi:10.1117/12.671410

Bohlin R. C., Dickinson M. E., Calzetti D., 2001, The Astronomical Journal, 122, 2118

Davis S. P., Abrams M. C., Brault J. W. J. W., 2001, Fourier transform spectrometry. Academic Press, San Diego

Drissen L., Bernier A.-P., Rousseau-Nepton L., Alarie A., Robert C., Joncas G., Thibault S., Grandmont F., 2010, in McLean I. S., Ramsay S. K., Takami H., eds, Vol. 7735, SPIE Astronomical Telescopes + Instrumentation. International
Society for Optics and Photonics, pp 77350B-77350B-10, doi:10.1117/12.856470

Foreman-Mackey D., 2016, The Journal of Open Source Software, 24

Foreman-Mackey D., Hogg D. W., Lang D., Goodman J., 2012, Publications of the Astronomical Society of Pacific, Volume 125, Issue 925, pp. 306-312 (2013)., 125, 306

Formisano V., et al., 2005, Planetary and Space Science, 53, 963 Grandmont F., 2003, in Proceedings of SPIE. SPIE, pp 392-401, doi: $10.1117 / 12.457339$

Griffin M. J., et al., 2010, Astronomy and Astrophysics, 518, L3

Guerrero M. A. M. A., Manchado A., Chu Y.-H., Uerrero M. A. G., Anchado A. M., Hu Y. C., 1997, The Astrophysical Journal, 487, 328

Harris F., 1978, Proceedings of the IEEE, 66, 51

Jones E., Oliphant T., Peterson P., et al., 2001, SciPy: Open source scientific tools for Python, http://www.scipy.org/

Kauppinen J., Partanen J., 2001, Fourier Transforms in Spectroscopy. Wiley-VCH

Kawada M., et al., 2008, Publications of the Astronomical Society of Japan

Kunde V. G., et al., 1996, Proc. SPIE Vol. 2803, 2803, 162

Levenberg K., 1944, Quarterly of Applied Mathematics, 2, 164

Luridiana V., Morisset C., Shaw R. A., 2012, in Planetary Nebulae: An Eye to the Future. IAU Symposium, pp 422-423, doi:10.1017/S1743921312011738

Maillard J., Michel G., 1982, Instrumentation for Astronomy with Large Optical Telescopes. Astrophysics and Space Science Library Vol. 92, Springer Netherlands, Dordrecht, doi:10.1007/978-94-009-7787-7

Marquardt D. W., 1963, Journal of the Society for Industrial and Applied Mathematics, 11, 431

Martin T., 2015, Phd thesis, Université Laval

Martin T., Drissen L., Joncas G., 2012, in Radziwill N. M., Chiozzi G., eds, Vol. 2, SPIE - Software and Cyberinfrastructure for Astronomy II. pp $84513 \mathrm{~K}-84513 \mathrm{~K}-9$, doi:10.1117/12.925420

Martin T., Drissen L., Joncas G., 2015, Astronomical Data Analysis Software an Systems XXIV (ADASS XXIV), 495

Medina S.-N. X., Arthur S. J., Henney W. J., Mellema G., Gazol a., 2014, Monthly Notices of the Royal Astronomical Society, 445,1797

Naylor D. A., Tahic M. K., 2007, Journal of the Optical Society of America A, 24, 3644

Naylor D. A., Gom B. G., Zhang B., 2006, in Zmuidzinas J., Holland W. S., Withington S., Duncan W. D., eds, Vol. 6275, Millimeter and Submillimeter Detectors and Instrumentation for Astronomy III. Edited by Zmuidzinas. pp 62751Z-62751Z11, doi:10.1117/12.670552

O'Dell C. R., Sabbadin F., Henney W. J., 2007, The Astronomical Journal, 134, 1679

O’Dell C. R., Ferland G. J., Henney W. J., Peimbert M., 2013a, The Astronomical Journal, 145, 92

O’Dell C. R., Ferland G. J., Henney W. J., Peimbert M., 2013b, The Astronomical Journal, 145, 93

O'dell C. R., 1991, in Fragmentation of Molecular Clouds and Star Formation. Springer Netherlands, Dordrecht, pp 476479, doi:10.1007/978-94-011-3384-5_76

Osterbrock D., Ferland G., 2006, Astrophysics of gaseous nebulae and active galactic nuclei. University Science Books, Sausalito

Ott S., 2010, Astronomical Data Analysis Software and Systems XIX (ADASS XIX), 434

Shaw R. A., Dufour R. J., 1995, Publications of the Astronomical Society of the Pacific, 107, 896

Wright S. a., Corradi R. L. M., Perinotto M., 2005, Astronomy and Astrophysics, 436, 9 


\section{APPENDIX A: DEMONSTRATION OF THE GAUSSIAN-SINC CONVOLUTION FORMULA}

Even if the convolution of a sinc and a Gaussian is a result that can easily be found in the literature, the demonstration of the function considered in this paper (see equation 11), which is not trivial, is not given anywhere. Since only some of the major steps can be found in the Herschel Common Science System (HCSS) documentation, we provide here the full demonstration for reference.

Starting from equation 8 and following the convolution theorem, the convolution of a sinc and a Gaussian $S G(\sigma)$ can be rewritten as the inverse Fourier transform of

\section{A1 Analytic form of the convolution of a sinc and a Gaussian}

the product of the Fourier transforms of the convoluted function which leads to

$S G(\sigma)=\int_{-\infty}^{+\infty} \hat{S}(x) \hat{\delta}(x) \hat{G}(x) e^{2 i \pi \sigma x} \mathrm{~d} x$.

Let's first write down the Fourier transform of the 3 components of $S G(\sigma)$ as they can be found, for example, in Kauppinen \& Partanen (2001):

$\hat{S}(x)=\pi \Delta w \Pi(x \pi \Delta w)=\left\{\begin{array}{ll}\pi \Delta w & \text { if }|x| \leq \frac{1}{2 \pi \Delta w} \\ 0 & \text { otherwise }\end{array}\right.$,

$\hat{\delta}(x)=e^{-2 i \pi \sigma_{0} x}$,

$\hat{G}(x)=\sqrt{2 \pi} \Delta \sigma e^{-2 \Delta \sigma^{2} \pi^{2} x^{2}}$.

It follows that

$$
\begin{aligned}
& S G(\sigma)=\sqrt{2 \pi} \Delta \sigma \pi \Delta w \\
& \quad \int_{-\infty}^{+\infty} \Pi(x \pi \Delta w) e^{-2 \Delta \sigma^{2} \pi^{2} x^{2}} e^{2 i \pi x\left(\sigma-\sigma_{0}\right)} \mathrm{d} x .
\end{aligned}
$$

The rectangular function $\Pi$ defines new boundaries to the integral and the Euler's formula permit to separate this integral into an even term (multiplied by a cos) and an odd term (multiplied by a sin)

$$
\begin{array}{r}
S G(\sigma)=\sqrt{2 \pi} \Delta \sigma \pi \Delta w\left[\int_{-\frac{1}{2 \pi \Delta w}}^{+\frac{1}{2 \pi \Delta w}} e^{-2 \Delta \sigma^{2} \pi^{2} x^{2}} \cos \left(2 \pi x\left(\sigma-\sigma_{0}\right)\right) \mathrm{d} x\right. \\
\left.+i \int_{-\frac{1}{2 \pi \Delta w}}^{+\frac{1}{2 \pi \Delta w}} e^{-2 \Delta \sigma^{2} \pi^{2} x^{2}} \sin \left(2 \pi x\left(\sigma-\sigma_{0}\right)\right) \mathrm{d} x\right] . \quad(\mathrm{A} 6)
\end{array}
$$

The integral of the odd term is equal to 0 and the integral of the even term is simply twice the integral along the positive part. Using once again Euler's formula we can split the even term into two exponential terms

$$
\begin{aligned}
S G(\sigma)=\sqrt{2 \pi} \Delta \sigma \pi \Delta w & {\left[\int_{0}^{\frac{1}{2 \pi \Delta w}} e^{2 i \pi x\left(\sigma-\sigma_{0}\right)-2 \Delta \sigma^{2} \pi^{2} x^{2}} \mathrm{~d} x\right.} \\
+ & \left.\int_{0}^{+\frac{1}{2 \pi \Delta \omega}} e^{-2 i \pi x\left(\sigma-\sigma_{0}\right)-2 \Delta \sigma^{2} \pi^{2} x^{2}} \mathrm{~d} x\right] .
\end{aligned}
$$

We can rearrange this equation by completing the square into both terms and rewrite it as

$$
\begin{array}{r}
S G(\sigma)=\sqrt{2 \pi} \Delta \sigma \pi \Delta w \exp \left(-\frac{\left(\sigma-\sigma_{0}\right)^{2}}{2 \Delta \sigma^{2}}\right)\left[\int_{0}^{z} e^{-\alpha(x+i \beta)^{2}} \mathrm{~d} x\right. \\
\left.+\int_{0}^{z} e^{-\alpha(x+i \beta)^{2}} \mathrm{~d} x\right] .
\end{array}
$$

where $\alpha=2 \Delta \sigma^{2} \pi^{2}, \beta=\frac{\sigma-\sigma_{0}}{2 \pi \Delta \sigma^{2}}$ and $z=\frac{1}{2 \pi \Delta w}$.

The first integral can be found by substituting $x$ with $u=x+i \beta$

$\int_{0}^{z} e^{-\alpha(x+i \beta)^{2}} \mathrm{~d} x=\int_{i b}^{z+i b} e^{-\alpha u^{2}} \mathrm{~d} u$
$=\int_{0}^{z+i b} e^{-\alpha u^{2}} \mathrm{~d} u-\int_{0}^{i b} e^{-\alpha u^{2}} \mathrm{~d} u$.

From the erf definition,

$\int_{0}^{z} e^{-a x^{2}}=\frac{\sqrt{\pi}}{2 \sqrt{a}} \operatorname{erf}(\sqrt{a} z)$,

the value of the first integral becomes,

$\int_{0}^{z} e^{-\alpha(x+i \beta)^{2}} \mathrm{~d} x=\frac{\sqrt{\pi}}{2 \sqrt{\alpha}}(\operatorname{erf}(\sqrt{\alpha}(z+i \beta))-\operatorname{erf}(i \sqrt{\alpha} \beta))$.

In an analogous fashion we can substitute $x$ with $u=x-i \beta$ in the second integral and rewrite it as

$$
\int_{0}^{z} e^{-\alpha(x-i \beta)^{2}} \mathrm{~d} x=\frac{\sqrt{\pi}}{2 \sqrt{\alpha}}(\operatorname{erf}(\sqrt{\alpha}(z-i \beta))+\operatorname{erf}(i \sqrt{\alpha} \beta)) .
$$

$S G(\sigma)$ is therefore

$$
\begin{array}{r}
S G(\sigma)=\frac{\pi \Delta w}{2} \exp \left(-\frac{\left(\sigma-\sigma_{0}\right)^{2}}{2 \Delta \sigma^{2}}\right)\left[\operatorname{erf}\left(\frac{\Delta \sigma}{\sqrt{2} \Delta w}+i \frac{\sigma-\sigma_{0}}{\sqrt{2} \Delta \sigma}\right)\right. \\
\left.+\operatorname{erf}\left(\frac{\Delta \sigma}{\sqrt{2} \Delta w}-i \frac{\sigma-\sigma_{0}}{\sqrt{2} \Delta \sigma}\right)\right] .
\end{array}
$$

\section{A2 Normalized formula}

Now we might want to normalize the above function so that its peak value (when $\sigma=\sigma_{0}$ ) is 1, i.e. $S G\left(\sigma_{0}\right)=1$. This translates to

$\pi \Delta w \operatorname{erf}\left(\frac{\Delta \sigma}{\sqrt{2} \Delta w}\right)=1$,

which permits to write the normalized convolution of a sinc and a Gaussian given in equation 11 as

$$
\begin{aligned}
S G(\sigma)=\frac{\exp \left(-\frac{\left(\sigma-\sigma_{0}\right)^{2}}{2 \Delta \sigma^{2}}\right)}{2 \operatorname{erf}\left(\frac{\Delta \sigma}{\sqrt{2} \Delta w}\right)}\left[\operatorname{erf}\left(\frac{\Delta \sigma}{\sqrt{2} \Delta w}+i \frac{\sigma-\sigma_{0}}{\sqrt{2} \Delta \sigma}\right)\right. \\
\left.+\operatorname{erf}\left(\frac{\Delta \sigma}{\sqrt{2} \Delta w}-i \frac{\sigma-\sigma_{0}}{\sqrt{2} \Delta \sigma}\right)\right] .
\end{aligned}
$$

\section{A3 Value of the integral on the whole space}

As $S(\sigma), \delta\left(\sigma-\sigma_{0}\right)$ and $G(\sigma)$ are integrable functions, the integral of $S G(\sigma)$ on the whole space can be computed easily if we consider, after the Fubini's theorem, that it is the 
product of the integrals of $S(\sigma), \delta\left(\sigma-\sigma_{0}\right)$ and $G(\sigma)$. In the case of its unnormalized version (see equation A14) it follows

$\int_{-\infty}^{+\infty} S G(\sigma)=\int_{-\infty}^{+\infty} S(\sigma) * \delta\left(\sigma-\sigma_{0}\right) * G(\sigma)$

$=\int_{-\infty}^{+\infty} S(\sigma) \times \int_{-\infty}^{+\infty} \delta\left(\sigma-\sigma_{0}\right) \times \int_{-\infty}^{+\infty} G(\sigma)$

$=\sqrt{2 \pi} \Delta \sigma \times \pi \Delta w$.

The integral of the normalized function (see equation A16) can be obtained by dividing this results by the normalization factor, therefore

$\int_{-\infty}^{+\infty} S G(\sigma)=\frac{\sqrt{2 \pi} \Delta \sigma}{\operatorname{erf}\left(\frac{\Delta \sigma}{\sqrt{2} \Delta w}\right)}$.

\section{APPENDIX B: ABSOLUTE VELOCITY CALIBRATION OF NGC 6720}

The only uncertainty in terms of velocity calibration of Fourier Transform spectra is the zero point. The velocity zero point can be estimated at each position in the field of view by observing a laser source with a known wavelength. SITELLE's wavelength calibration is based on a high-resolution laser cube taken with the telescope pointing at zenith. As most of the observations are made with a different gravity vector, the whole optical structure is subject to a certain amount of distortion which changes the pixelto-pixel zero point. Also, the wavelength of the laser is expected to have a long-term drift. A more refined wavelength calibration must therefore be made by fitting the numerous Meinel $\mathrm{OH}$ bands present in the filter passband. A velocity calibration map can then be reconstructed from the $\mathrm{OH}$ lines velocity measured on integrated spectra that are taken all over the field. The whole process has been implemented in ORCS (Martin et al. 2016, in preparation). The sky velocity map used to calibrate NGC 6720 and an example of a fit realized on one of the integrated sky spectra are shown in Fig. B1. The uncertainty on sky lines velocity in the integrated spectra is around $1 \mathrm{~km} \mathrm{~s}^{-1}$. An overall uncertainty on the pixel-to-pixel calibration of $0.5 \mathrm{~km} \mathrm{~s}^{-1}$ appears to be a conservative estimation.

\section{APPENDIX C: DISTRIBUTION OF THE POSTERIOR PROBABILITY FOR A FIT ON A SPECTRUM OF NGC 6720}

Fig. C1 shows the marginalized distribution of the posterior probability for each parameter of a fit realized on the spectrum shown in Fig. 7 with a Markov-Chain MonteCarlo algorithm. The fitted model is the sum of 5 lines $(\mathrm{H} \alpha$, $[\mathrm{N}$ II] $\lambda 6548,[\mathrm{~N}$ II] $\lambda 6584$, [S II] $\lambda 6717$, [S II] $\lambda 6731)$ plus a constant continuum (not shown here). Line model is the convolution of a sinc line and a Gaussian broadening as described in Section 2.2. [N $\mathrm{NI}]$ and $[\mathrm{S} \mathrm{II}]$ lines are sharing the same velocity parameter and the same broadening parameter while the $\mathrm{H} \alpha$ line as its own velocity and broadening. Amplitudes are fitted independently. FWHM is a fixed parameter which depends only on the number of steps, i.e. on the resolution (see Section 2.1). The diagonal of this corner plot shows the marginalized distribution of the posterior probability for each parameter independently. The other panels shows the two-dimensional marginalized distribution for all couple of parameters. This corner plot has been realized with the Python library corner.py (Foreman-Mackey 2016).

\section{APPENDIX D: UNCERTAINTY ON THE DATA}

Fig. D1 and D2 show the uncertainty on the maps shown respectively in Fig. 5 and 8 . The order of the panels is the same. The uncertainty is calculated from the diagonal of the covariance matrix returned by the function scipy.optimize.curve_fit which implements the Levenberg-Marquardt minimization algorithm (Jones et al. 2001).

This paper has been typeset from a $\mathrm{T}_{\mathrm{E}} \mathrm{X} / \mathrm{AAT}_{\mathrm{E}} \mathrm{X}$ file prepared by the author. 

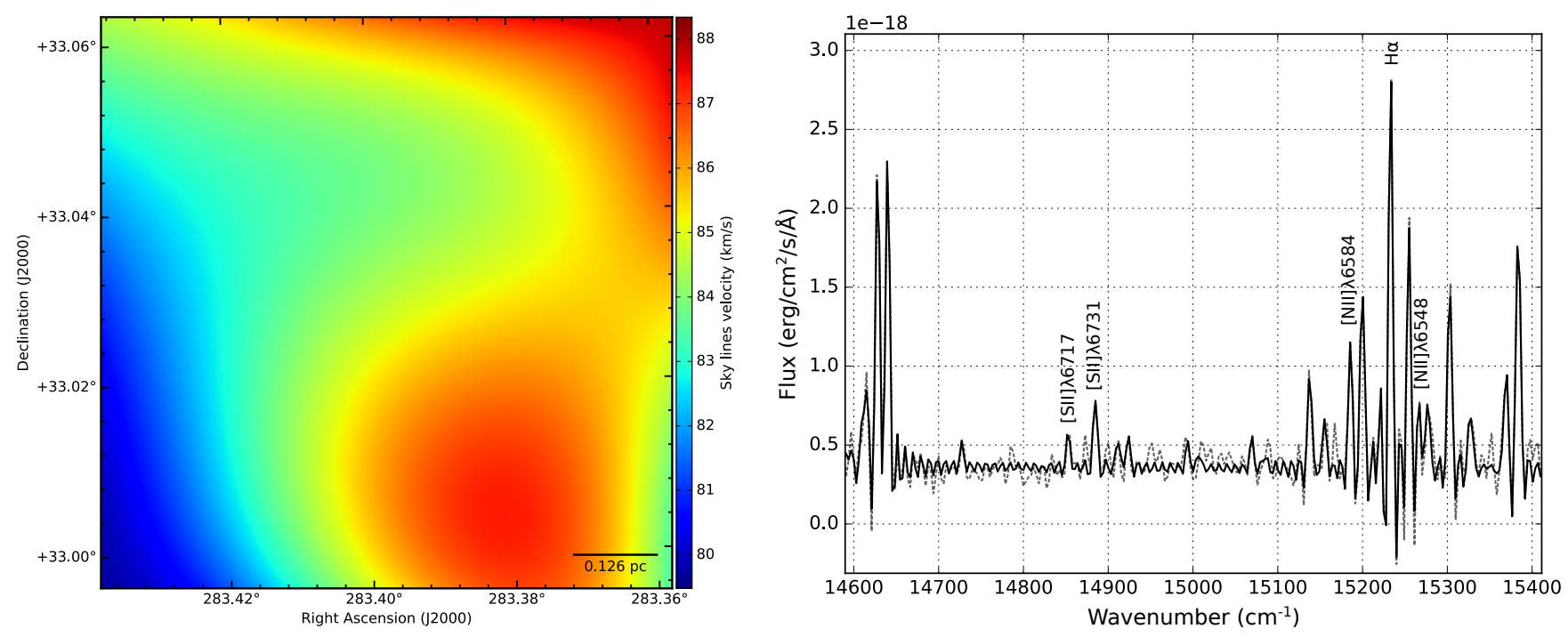

Figure B1. Left: Map of the measured sky lines velocity used to calibrate the cube of NGC 6720. Right: Example of a fit realized on one of the integrated sky spectra of NGC 6720 that have been used to reconstruct the velocity calibration map shown in the left panel. Some diffuse emission of the interstellar medium surrounding the nebula can be seen in the spectrum. The position of these lines, which have been fitted with a different velocity parameter, is indicated. See text for details. 


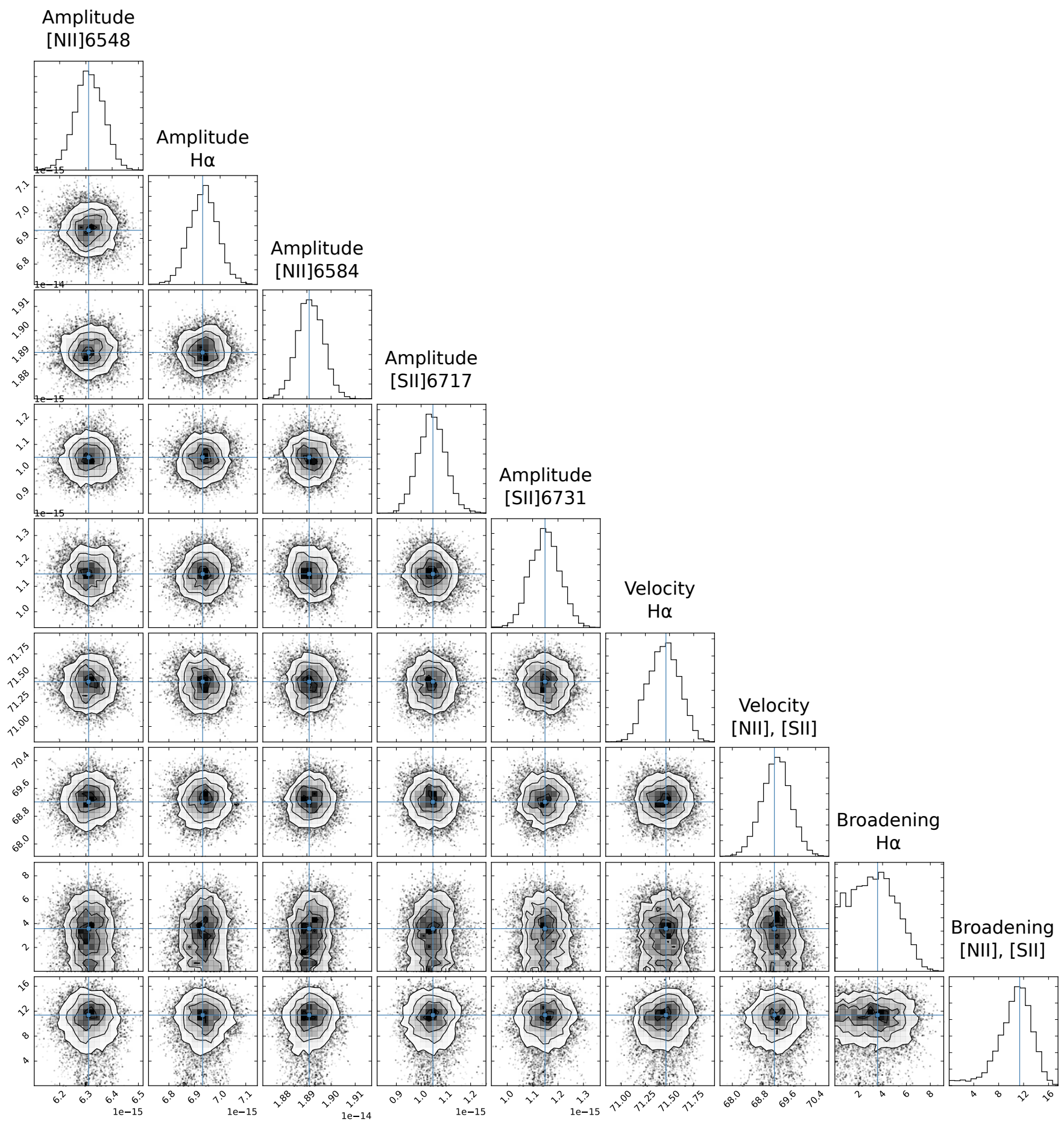

Figure C1. Corner plot of the marginalized distribution of the posterior probability for each parameter of a fit realized on the spectrum shown in Fig. 7 with a Markov-Chain Monte-Carlo algorithm. See text for details. 

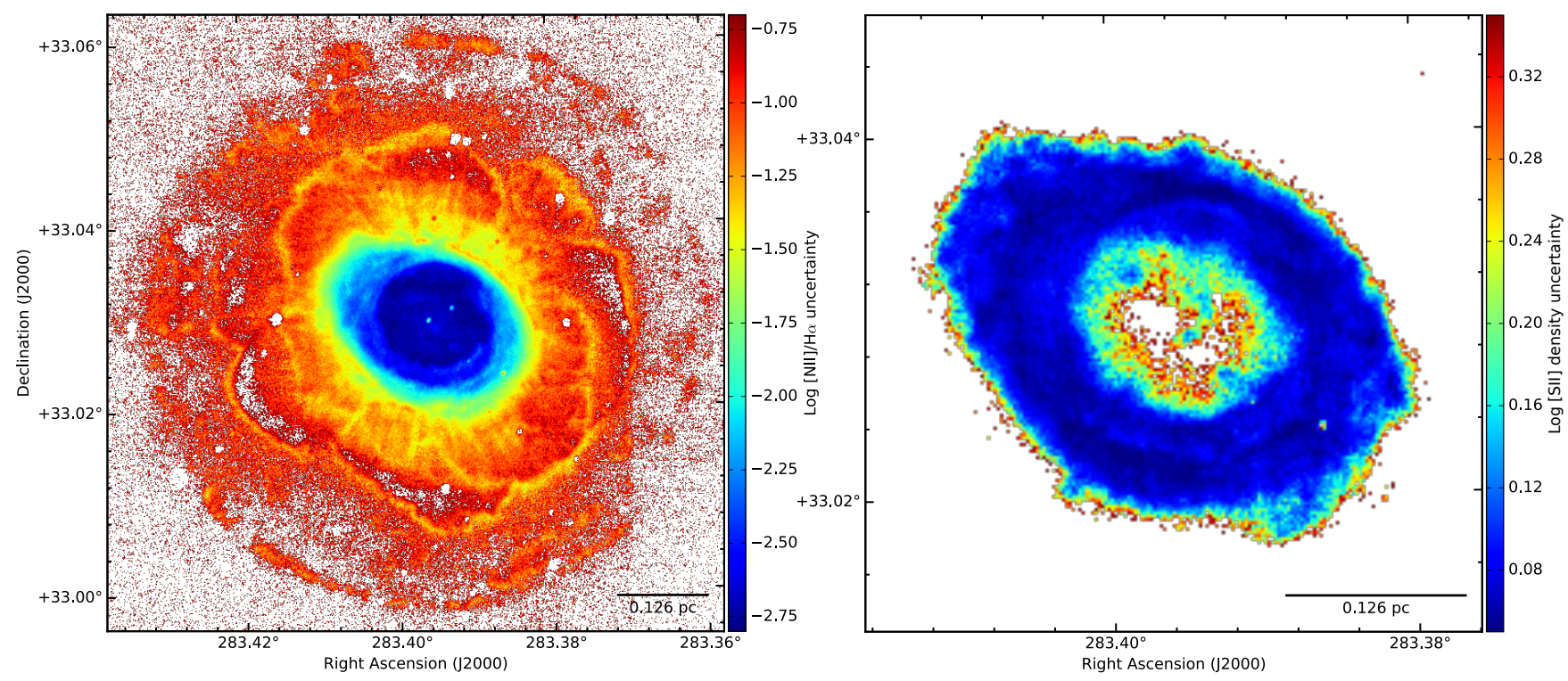

Figure D1. Uncertainty of the maps shown in Fig. 5. The order of the panels is the same. See text for details. 

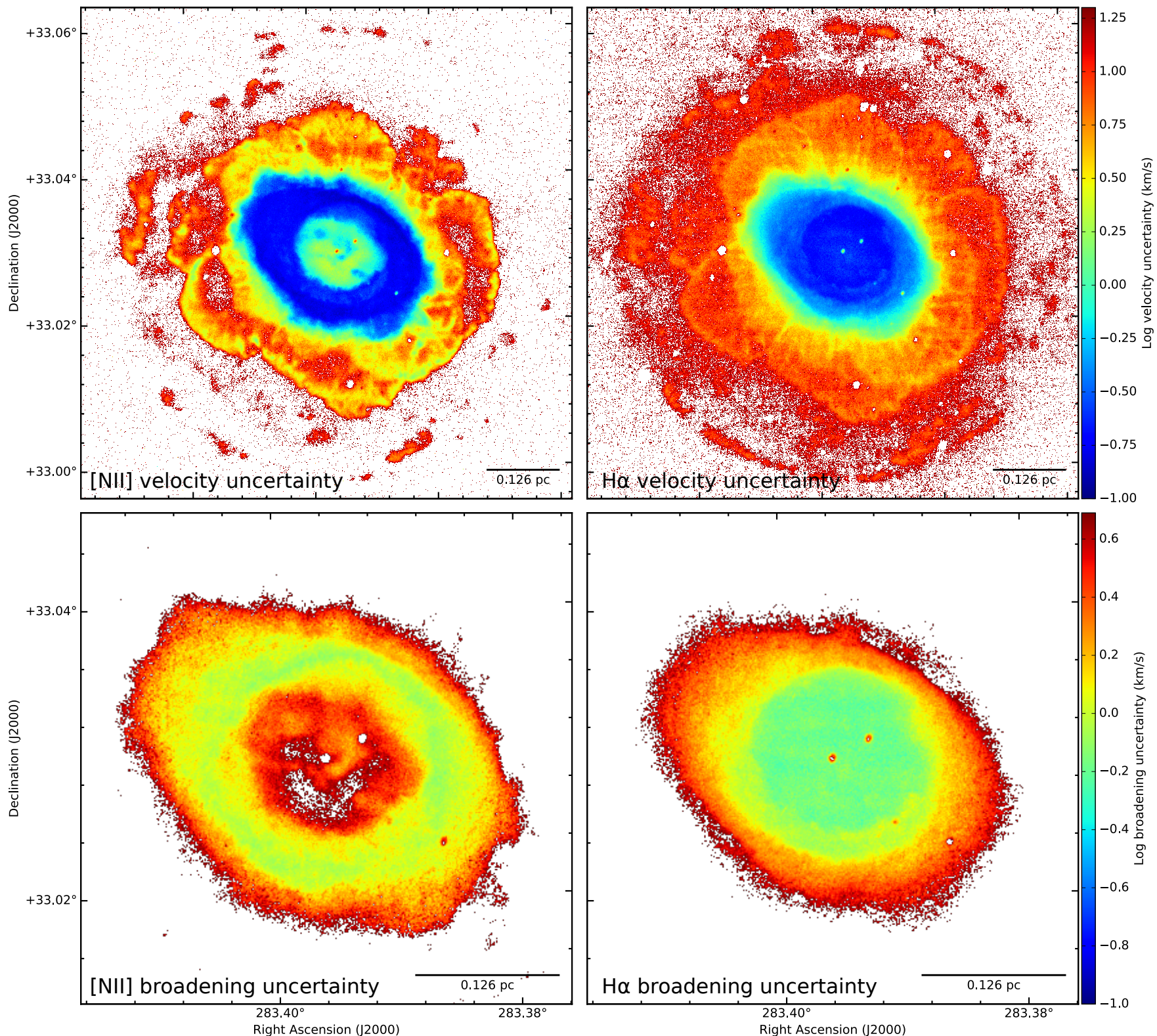

Figure D2. Uncertainty of the maps shown in Fig. 8. The order of the panels is the same. See text for details. 